\title{
Long-Term Expansion Planning of the Transmission Network in India under Multi-Dimensional Uncertainty
}

\author{
Spyros Giannelos *, Anjali Jain (D), Stefan Borozan (D), Paola Falugi, Alexandre Moreira, Rohit Bhakar (D, \\ Jyotirmay Mathur and Goran Strbac
}

Department of Electrical and Electronic Engineering, Imperial College London, London SW7 2AZ, UK; 2017ren9505@mnit.ac.in (A.J.); s.borozan@imperial.ac.uk (S.B.); p.falugi@imperial.ac.uk (P.F.); a.moreira14@imperial.ac.uk (A.M.); rbhakar.ee@mnit.ac.in (R.B.); jmathur.mech@mnit.ac.in (J.M.); g.strbac@imperial.ac.uk (G.S.)

* Correspondence: s.giannelos@imperial.ac.uk

check for updates

Citation: Giannelos, S.; Jain, A.; Borozan, S.; Falugi, P.; Moreira, A.; Bhakar, R.; Mathur, J.; Strbac, G. Long-Term Expansion Planning of the Transmission Network in India under Multi-Dimensional Uncertainty. Energies 2021, 14, 7813. https:// doi.org/10.3390/en14227813

Academic Editor: Jürgen Heinz Werner

Received: 5 September 2021

Accepted: 19 November 2021

Published: 22 November 2021

Publisher's Note: MDPI stays neutral with regard to jurisdictional claims in published maps and institutional affiliations.

Copyright: (c) 2021 by the authors. Licensee MDPI, Basel, Switzerland. This article is an open access article distributed under the terms and conditions of the Creative Commons Attribution (CC BY) license (https:// creativecommons.org/licenses/by/ $4.0 /)$.
Abstract: Considerable investment in India's electricity system may be required in the coming decades in order to help accommodate the expected increase of renewables capacity as part of the country's commitment to decarbonize its energy sector. In addition, electricity demand is geared to significantly increase due to the ongoing electrification of the transport sector, the growing population, and the improving economy. However, the multi-dimensional uncertainty surrounding these aspects gives rise to the prospect of stranded investments and underutilized network assets, rendering investment decision making challenging for network planners. In this work, a stochastic optimization model is applied to the transmission network in India to identify the optimal expansion strategy in the period from 2020 until 2060, considering conventional network reinforcements as well as energy storage investments. An advanced Nested Benders decomposition algorithm was used to overcome the complexity of the multistage stochastic optimization problem. The model additionally considers the uncertainty around the future investment cost of energy storage. The case study shows that deployment of energy storage is expected on a wide scale across India as it provides a range of benefits, including strategic investment flexibility and increased output from renewables, thereby reducing total expected system costs; this economic benefit of planning with energy storage under uncertainty is quantified as Option Value and is found to be in excess of GBP $12.9 \mathrm{bn}$. The key message of this work is that under potential high integration of wind and solar in India, there is significant economic benefit associated with the wide-scale deployment of storage in the system.

Keywords: energy storage; flexibility; India; nested benders decomposition; network planning; option value; stochastic optimization

\section{Introduction}

India is undergoing a large-scale transformation of its economy as part of the global effort to tackle climate change. This is a topic that is very high on the agenda for the Indian government as recent reports have provided evidence that there has been an increase of about $0.7^{\circ} \mathrm{C}$ in average temperature over India for the period from 1971 to 2018 [1]. In this context, the country has committed towards achieving greater levels of decarbonization of its energy sector, as also demonstrated by its ratification of the Paris Convention on Climate Change [2].

The country's energy mix continues to be dominated by conventional energy sources, and mainly by coal, which is the cheapest and the most abundant energy resource in the country. For instance, the state-owned company Coal India is the largest coal mining company in the world reflecting the fact that coal is a major source of revenue for all states in India and it still provides around $70 \%$ of its electricity output. In fact, coal accounted for around $75 \%$ of the country's electricity generation in the year 2018 [3]. This is the primary reason why India ranks among the top three carbon dioxide emitters in the world. 
In India, the electricity sector alone contributes almost $45 \%$ of total $\mathrm{CO}_{2}$ emissions. This sector is being fundamentally transformed with steadily increasing electrical demand across other sectors, including agriculture, transport, industry, commercial and residential. Electricity is becoming more and more accessible to the wider population. For instance, around 700 million people in India gained access to electricity between 2000 and 2018 and the government has recently stated that $100 \%$ of its villages have been connected to the electricity grid [4]. In addition, the government has announced investment plans aiming to significantly reduce the frequency of power outages [5]. In this context, it is expected that the country's continued economic and population growth, as well as its fundamental electrification, will lead to higher levels of electricity demand in the near future [6].

Moreover, the integration of renewable energy has been exhibiting an impressive rise. By early 2020, around $84 \mathrm{GW}$, out of $366 \mathrm{GW}$ total installed generation capacity, were grid-connected renewable electricity capacity. Furthermore, the government of India has committed towards having installed $175 \mathrm{GW}$ of renewables by the year 2022 [7], including $100 \mathrm{GW}$ from solar PV, $60 \mathrm{GW}$ from wind onshore and offshore, $10 \mathrm{GW}$ from biomass and $5 \mathrm{GW}$ from small hydroelectric power units. Another commitment is for the country to have $450 \mathrm{GW}$ of renewable capacity by the year 2030. In fact, India is already making notable progress; for instance, its per capita emissions have decreased to 1.6 tonnes of $\mathrm{CO}_{2}$, which is well below the worldwide average of 4.4 tonnes [8].

This transition towards a low-carbon energy sector may create significant challenges for the safe operation of the country's electricity system in the future. These challenges are related to potentially increased power flows resulting from the higher levels of electricity demand and electricity generation as well as from the presence of smart grid technologies that tend to lead to increased asset utilization [9]. For instance, based on current policies, India's energy demand may double by 2040, as a result of increased appliance ownership and cooling needs [10], which may place significant stress on the grid.

In addition, another significant challenge is associated with the increased uncertainty around the timing (i.e., "when"), magnitude (i.e., "how much"), and location (i.e., "where") of connections of new demand and generation capacity since their future deployment patterns are not known a priori with certainty to the network planner. The presence of this uncertainty can give rise to the prospect of inefficient investments and stranded, or underutilized, assets because it may render network planners unable to make investment decisions with sufficient foresight.

Therefore, in order to successfully address the aforementioned challenges, which is vital for maintaining a secure supply of electricity well into the future, a considerable amount of investment in network reinforcement may be required over the coming decades [3]. However, the aforementioned uncertainty involves the risk that much of the eventual network reinforcement may end up stranded or underutilized, which may lead to high associated transition costs. In this context, smart grid technologies, such as energy storage, can constitute an option that can provide the required strategic flexibility, which will enable the network planner to hedge against the risk of stranded conventional assets by allowing the displacement and/or the deferral of large capital commitments on a conditional basis until the realization of a scenario suggests that they would be economically justified. This leads to the definition of the Option Value, which is the value of the total flexibility of energy storage, under the presence of uncertainty, and can be quantified as the net economic benefit accrued from its deployment in the system [11-13].

Energy storage integration has been shown to provide significant benefits both in distribution and transmission networks. For example, references [14,15] demonstrate that distribution network planning with energy storage can support higher renewables integration as well as cost reductions for local energy communities and clusters. In transmission, reference [16], applied in the context of India specifically, demonstrates that the large-scale deployment of energy storage technologies could indeed facilitate renewables integration and support a low carbon emission intensity of the Indian power sector. The authors apply a multi-region approach for planning the cost-optimal allocation of the storage technology 
mix as well as conventional transmission reinforcements to support the integration of renewables up to the year 2040. However, the proposed MILP model is deterministic and does not consider the impact of future uncertainties. In addition, a multistage stochastic optimization framework is the most appropriate method for option valuation of smart technologies in the context of investment decision making in energy infrastructure [17]. Therefore, the application of a state-of-the-art network expansion planning framework is essential in handling the impacts of uncertainty and capturing the full value of energy storage investments.

In this context, this paper presents an advanced approach based on stochastic optimization that quantifies the Option Value of energy storage and gives insights into the investment requirements for a secure and cost-effective expansion of the transmission network in India. Therefore, the contributions of the current work can be summarized as follows.

- Quantification of the Option Value of investing in energy storage across India, covering the period 2020-2060.

- Presentation of key investment insights and notable conclusions for India's electricity system covering the period 2020-2060.

- Application of an advanced decomposition approach to overcome the increased complexity related to the application of stochastic optimization under multi-dimensional uncertainty, to the India context.

The paper is structured in the following manner. Section 2 presents the literature review on energy storage and Option Value methodologies for smart grid technologies. In Section 3, the mathematical problem formulation and solution methodology are explained, and the associated mathematical formulation is presented. Section 4 presents the description of the case study and the discussion of findings, while Section 5 presents future work pathways and concludes.

\section{Energy Storage and Option Value in the India Context}

Wide-scale deployment of energy storage in India's power system may offer significant techno-economic benefits and contribute to the cost-effective integration of the large amount of planned renewable capacity. Specifically, one key benefit that energy storage can provide is strategic flexibility that can enable the network planner to hedge against the risks associated with the presence of both exogenous and endogenous sources of uncertainty. Through the deployment of energy storage, the planner obtains the "real" option to delay/defer or displace/avoid undertaking conventional investments. In this case, when there is at least one source of uncertainty considered in the model, the economic total net benefit accrued from the deployment of this technology is known as Option Value of energy storage [11,12].

In addition, energy storage can offer additional benefits including support for the real-time balancing of electricity supply and demand through the provision of ancillary services [18-20], power quality improvements [21] decentralized coordination of distributed energy resources within microgrids [22], provision of secure electricity supply to consumers through reduction of peak demand [23], and increased output from renewables (reduced output curtailment) since it can store the electricity produced during sunny/windy periods and feed it back to the grid at another time.

These benefits can help India establish a future-proof energy infrastructure, with large capacity of renewables and increased electricity demand, despite the possible large amount of uncertainty in the system development [9]. In this context, it is forecasted that India will account for more than one-third of the total deployment of battery storage technology, globally, by the year 2040 [24] and that the country will need more capacity of it than any other country in the world over the coming decades. Unlike pumped hydro storage, battery storage is location-independent and has been seeing considerable cost reductions and technological improvements on a global scale. 
Despite the considerable techno-economic benefits that energy storage can provide, the current presence of market and regulatory barriers in India may constitute a barrier in the extensive deployment of energy storage with implications for a future power system's ability to reliably accommodate large amounts of renewables. Particularly, the existing regulatory and policy framework lacks planning standards that provide an explicit and formal methodological framework for the assessment of the aforementioned energy storage benefits. Hence, an update of the planning standards is of fundamental significance so that the full range of benefits of this technology can be properly taken into consideration. For instance, the quantification of the Option Value of energy storage through a stochastic optimization framework is a task that has not ever been conducted in the past, risking the potential under-evaluation of energy storage as a candidate investment technology. Thus, a clearly defined asset valuation framework for each possible technology that can be deployed in the electricity grid of India will be the basis for the establishment of a robust level playing field. The adopted approach can be fundamental for optimal investment decision making in India's electricity infrastructure over the coming decades.

In this context, the present paper presents a methodology for the quantification of the Option Value of energy storage in India. This methodology includes the following steps. First, it involves modelling energy storage operation and investment within a stochastic optimization framework that considers the system expansion and operation in a multistage formulation. Secondly, the stochastic optimization model is solved with and without the consideration of energy storage. Finally, the difference in total expected system cost is equal to the Option Value of energy storage, reflecting the total net economic benefit that the planner accrues when energy storage is available for investment.

This methodology is characterized by simplicity and clarity as it is easy to explain to decision makers, and it is also implementable not only for evaluating the Option Value of storage but of any other smart grid technology, such as soft open points, coordinated voltage control, and demand side response [25]. On the other hand, other methodologies of asset valuation that do not make use of mathematical optimization, such as Real Options analysis [26,27], may not be suitable for optimal investment decision making in energy infrastructure since their application scope is restricted to a small number of candidate investment strategies defined a priori, whereas in reality, a very large number of combinations of investment technologies exists, due to multiple stages, locations, capacities, and types of technologies considered in the problem, which render the use of optimization necessary for obtaining the optimal solution.

\section{Planning Model Formulation and Solution Procedure}

Expansion planning models for power systems can be basically divided into two categories. The first category is called static expansion planning [20,28-30]. In static planning, the system planner considers a target year and investment decisions can be made only once to address a particular objective such as accommodating an increase in demand. The second category is named dynamic expansion planning [31]. In dynamic planning, the system planner needs to determine the expansion scheme over a longer horizon by taking investment decisions multiple times throughout this horizon while coping with partial uncertainty realizations. In this paper, a methodology is presented for dynamic expansion planning.

The previous sections described the multiple benefits of energy storage and the steps of the methodology for quantifying its Option Value. According to this methodology, the first step is to incorporate the modelling of energy storage within a stochastic optimization framework. Furthermore, the high complexity associated with optimal investment decision making requires the implementation of an advanced decomposition methodology. Accordingly, Section 3.1 explains the need for and type of decomposition method applied in the context of planning the future high voltage transmission network of India, then Section 3.2 presents the mathematical formulation of the stochastic optimization problem and Section 3.3 explains the solution algorithm. 


\subsection{Advanced Decompostion of the Large-Scale Transmission Planning Problem}

There are several factors contributing to the complexity of the proposed optimization problem. Firstly, the high voltage transmission network of India, presented in Section 4, is of relatively large size leading to many possible investment candidates. Secondly, the multistage formulation of the problem, i.e., the ability to invest in multiple instances throughout the planning horizon, leads to multiple decision points to optimize. Finally, the ability to invest in different alternatives, namely line reinforcement and energy storage, introduces further decision variables, as well as additional inter-temporal modelling complexities. In order to solve this large-scale problem, the implementation of an advanced decomposition method is required. Reference [31] proposes a novel temporal decomposition technique based on Nested Benders decomposition and it proves its superior performance against other methods in the context of network expansion planning. As such, it suits the purpose of the present research.

The stochastic expansion planning objective minimizes the overall system expected cost across the scenario tree subject to investment and operational constraints.

The original multistage stochastic problem is decomposed into several two-stage problems. A master problem $\left(\mathbb{P}_{m}^{M}\right)$ and a subproblem $\left(\mathbb{P}_{m}^{S}\right)$ are formulated for each scenariotree node $m$. The formulation of $\mathbb{P}_{m}^{M}$ follows the equations in Section 3.2, whereas the problems $\mathbb{P}_{m}^{S}$ are a relaxed version of the master problem. The relaxation is necessary in order to avoid non-convexity issues due to the presence of binary variables.

Note that the available investment options in the presented framework have different construction and commissioning times, therefore the delay between the decision to invest and the asset becoming operational is different for line reinforcements and energy storage. This requires the inclusion of non-sequential state equations that link decision variables and constraints across all stages in the multistage formulation, which prevents the straightforward application of a temporal decomposition scheme. In order to apply the advanced temporal decomposition method, auxiliary state variables are introduced that carry information on investments and their operational status across different states. The definition of these variables and the corresponding reformulation of non-sequential state equations for the application in the decomposed planning problem are presented in [31].

\subsection{Mathematical Formulation}

As mentioned in Section 2, the planning framework aims to propose the optimal long-term network expansion strategy under multi-dimensional uncertainty from a very large number of investment possibilities considering the type, size, location, and timing of investments. As such, the stochastic problem involves a very large number of continuous and binary decision variables and is, therefore, formulated as a mixed-integer linear programming problem. Under the applied temporal decomposition method, a separate optimization problem is formulated for each scenario tree node $m$, described by Equations (1)-(40).

The present temporal decomposition links decision variables at a given stage to variables in the previous stage only, and it extends the formulation in [31] to include lifespan of candidate storage technologies. To characterize when investment and commissioning of the candidate technologies are available, information on their building-delays and lifespan needs to be used. For this purpose, let $\gamma_{\max }^{L}(\omega) \triangleq \max _{l}\left(\max \left\{1, \gamma_{l, \omega}\right\}\right), \omega \in$ $\Omega_{W}, \gamma_{\text {max }}^{S}(h) \triangleq \max \left\{1, \gamma_{h}\right\}$, and $\gamma_{\text {max }}^{S^{R}}(h) \triangleq \max \left\{1, \gamma_{h}+\delta_{h}\right\}, \forall h \in \Omega_{H}^{T}$. Note that, as explained in the previous subsection, the formulation of the master problems $\mathbb{P}_{m}^{M}$ follows the presented formulation, while in the subproblems $\mathbb{P}_{m}^{S}$ all binary variables are relaxed.

$$
\min _{x_{m}, \alpha_{j}^{m}}\left\{V_{m}\left(x_{m}\right)+\sum_{j \in \mathcal{N}^{+}(m)} \alpha_{j}^{m}\right\}
$$

where

$$
V_{m}=\pi_{m}\left(V_{m}^{I}+r_{\epsilon(m)}^{O} V_{m}^{O}\right)
$$




$$
\begin{aligned}
& V_{m}^{I}=\sum_{\forall l \in \Omega_{L}} \sum_{\forall \omega \in \Omega_{W}} r_{\epsilon(m)}^{L}\left(\kappa_{l, \omega}^{L, v} F_{m, l, \omega}^{I}+\kappa_{l, \omega}^{L, f} \beta_{m, l, \omega}^{I}\right)+\sum_{\forall h \in \Omega_{H}^{T}} r_{h, \epsilon(m)}^{S} \kappa_{m, h}^{S, v} S_{m, h}^{I} \\
& V_{m}^{O}=\sum_{\forall b \in \Omega_{B}} \mathrm{~W}_{b} \sum_{\forall t \in \Omega_{T}^{b}} \tau\left[\sum_{\forall g \in \Omega_{G}} c_{g} P_{m, t, g}+\sum_{\forall n \in \Omega_{N}} c_{V o L L} d_{m, t, n}\right] \\
& \text { subject to } \\
& \beta_{m, l, \omega}^{I} \in\{0,1\}, \forall l \in \Omega_{L}, \omega \in \Omega_{W} \\
& x_{m, l, \omega}^{\beta}=y_{p(m), l, \omega}^{\beta}:\left(\lambda_{m, l, \omega}^{\beta}\right), \forall l \in \Omega_{L}, \omega \in \Omega_{W} \\
& x_{m, l, \omega}^{c}=y_{p(m), l, \omega}^{c}:\left(\lambda_{m, l, \omega}^{c}\right), \forall l \in \Omega_{L}, \omega \in \Omega_{W} \\
& x_{m, l, \omega}^{F}=y_{p(m), l, i, \omega}^{F}:\left(\lambda_{m, l, i, \omega}^{c}\right), \forall l \in \Omega_{L}, i=1, \ldots, \gamma_{\text {max }}^{L}(\omega), \forall \omega \in \Omega_{W}, \\
& x_{m, h, i}^{S}=y_{p(m), h, i}^{S}:\left(\lambda_{m, h, i}^{S}\right), i=1, \ldots, \gamma_{\max }^{S}(h), \forall h \in \Omega_{H}^{T} \\
& x_{m, h, i}^{S^{R}}=y_{p(m), h, i}^{S^{R}}:\left(\lambda_{m, h, i}^{S^{R}}\right), i=1, \ldots, \gamma_{\text {max }}^{S^{R}}(h), \forall h \in \Omega_{H}^{T} \\
& \sum_{\forall \omega \in \Omega_{W}}\left[x_{m, l, \omega}^{\beta}+\beta_{m, l, \omega}^{I}\right] \leq 1, \forall l \in \Omega_{L} \\
& y_{m, l, \omega}^{\beta}=x_{m, l, \omega}^{\beta}+\beta_{m, l, \omega}^{I}, \forall l \in \Omega_{L}, \omega \in \Omega_{W} \\
& x_{m, l, \omega}^{\mathcal{c}}+\mathcal{F}_{l, \omega}^{\max } \beta_{m, l, \omega}^{I}-F_{m, l, \omega}^{I} \geq 0, \forall l \in \Omega_{L}, \omega \in \Omega_{W} \\
& y_{m, l, \omega}^{c}=x_{m, l, \omega}^{c}+\mathcal{F}_{l, \omega}^{\max } \beta_{m, l, \omega}^{I}-F_{m, l, \omega}^{I}, \forall l \in \Omega_{L}, \omega \in \Omega_{W} \\
& \widetilde{F}_{m, l}^{I}=\sum_{\forall \omega \in \Omega_{W}}\left[x_{m, l, 1, \omega}^{F}+B_{l, \omega}^{L, 0} F_{m, l, \omega}^{I}\right], \forall l \in \Omega_{L} \\
& y_{m, l, i, \omega}^{F}=\sum_{j=1}^{\gamma_{\text {max }}^{L}} A_{l, i, j, \omega}^{L} x_{m, l, j, \omega}^{F}+B_{l, i, \omega}^{L} F_{m, l, \omega}^{I}, \forall l \in \Omega_{L}, \omega \in \Omega_{W}, i=1, \ldots, \gamma_{\text {max }}^{L}(\omega) \\
& \widetilde{S}_{m, h}^{I} \leq \hat{S}_{h}, \forall h \in \Omega_{H}^{T} \\
& \widetilde{S}_{m, h}^{I}=x_{m, h, 1}^{S}-x_{m, h, 1}^{S^{R}}+B_{h}^{S, 0} S_{m, h}^{I}, \forall h \in \Omega_{H}^{T} \\
& y_{m, h, i}^{S}=\sum_{j=1}^{\gamma_{\max }^{S}} A_{h, i, j}^{S} x_{m, h, j}^{S}+B_{h, i}^{S} S_{m, h}^{I}, i=1, \ldots, \gamma_{\max }^{S}(h), \forall h \in \Omega_{H}^{T} \\
& y_{m, h, i}^{S^{R}}=\sum_{j=1}^{\gamma_{\max }^{S}} A_{h, i, j}^{S^{R}} x_{m, h, j}^{S^{R}}+B_{h, i}^{S^{R}} S_{m, h}^{I}, i=1, \ldots, \gamma_{\max }^{S^{R}}(h), \forall h \in \Omega_{H}^{T} \\
& 0 \leq P_{m, t, g} \leq \bar{P}_{m, t, g}, \forall g \in \Omega_{g} \backslash\left\{\underset{d \in\{r r, r e s\}}{\cup} \Omega_{G}^{H_{d}}\right\}, \forall t \in \Omega_{T}^{b}, \forall b \in \Omega_{B} \\
& P_{m, t-1, g}-\tau R D_{g} \leq P_{m, t, g} \leq P_{m, t-1, g}+\tau R U_{g}, g \in \Omega_{G} \backslash\left\{\underset{d \in\{r r, r e s\}}{\cup} \Omega_{G}^{H_{d}}\right\}, \forall t \in \Omega_{T}^{b} \backslash\left\{t_{b}^{1}\right\}, \forall b \in \Omega_{B} \\
& \underline{E}_{t, g}^{H_{d}} \leq E_{m, t, g}^{H_{d}} \leq \bar{E}_{t, g}^{H_{d}}, \forall g \in \Omega_{G}^{H_{d}}, \forall t \in \Omega_{T}^{b}, \forall b \in \Omega_{B}, d \in\{r r, r e s\} \\
& E_{m, T_{b}, g}^{H_{d}}=E_{m, t_{b}^{0}, g}^{H_{d}}, \forall g \in \Omega_{G}^{H_{d}}, d \in\{r r, r e s\}, \forall b \in \Omega_{B} \\
& E_{m, t_{b}^{1}, g}^{H_{d}}=E_{m, t_{b}^{0}, g}^{H_{d}}+\tau P_{t_{b}^{1}, g}^{H_{d,} \text { in }}-\tau P_{m, t_{b}^{1}, g}^{H_{d}}-\tau P_{m, t_{b}^{1}, g}^{H_{d, \text { shed }}}, \quad \forall g \in \Omega_{G}^{H_{d}}, d \in\{r r, r e s\}, \forall b \in \Omega_{B} \\
& E_{m, t, g}^{H_{d}}=E_{m, t-1, g}^{H_{d}}+\tau P_{t, g}^{H_{d,} \text { in }}-\tau P_{m, t, g}^{H_{d}}-\tau P_{m, t, g}^{H_{d,} \text { shed }}, \forall g \in \Omega_{G}^{H_{d}}, \forall t \in \Omega_{T}^{b} \backslash\left\{t_{b}^{1}\right\}, \forall b \in \Omega_{B} \\
& 0 \leq P_{m, t, g}^{H_{d}} \leq \bar{P}_{g}^{H_{d}}, \forall g \in \Omega_{G}^{H_{d}}, d \in\{r r, r e s\}, \forall t \in \Omega_{T}^{b}, \forall b \in \Omega_{B}
\end{aligned}
$$




$$
\begin{aligned}
& P_{m, t, g}=P_{m, t, g^{\prime}}^{H_{d}} \quad \forall g \in \Omega_{G}^{H_{d}} \cap \Omega_{G}, d \in\{r r, r e s\} \forall t \in \Omega_{T}^{b}, \forall b \in \Omega_{B} \\
& f_{m, t, l}=\frac{\theta_{m, t, u_{l}}-\theta_{m, t, v_{l}}}{X_{l}}, \forall l \in \Omega_{L}^{A C} \\
& -\left(\widetilde{F}_{m, l}^{I}+F_{l}^{0}\right) \leq f_{m, t, l} \leq\left(\widetilde{F}_{m, l}^{I}+F_{l}^{0}\right), \forall l \in \Omega_{L}, \forall t \in \Omega_{T}^{b}, \forall b \in \Omega_{B} \\
& \sum_{\forall g \in \Omega_{G}} I_{n, g} P_{m, t, g}-\sum_{\forall l \in \Omega_{L}} J_{n, l} f_{m, t, l}-\sum_{\forall h \in \Omega_{H}} H_{n, h}\left(P_{m, t, h}^{c}-P_{m, t, h}^{d}\right)+d_{m, t, n}=D_{m, t, n}^{b}, \forall t \in \Omega_{t}^{b}, \forall b \in \Omega_{B}, \forall n \in \Omega_{N} \\
& \sigma_{m, T_{b}, h}=\sigma_{m, t_{b}, h^{\prime}} \forall h \in \Omega_{H} \\
& \sigma_{m, t_{b}^{1}, h}=\sigma_{m, t_{b}^{0}, h}+\tau\left(\eta_{h}^{c} P_{m, t_{b}^{1}, h}^{c}-\frac{P_{m, t_{t}^{1}, h}^{d}}{\eta_{h}^{d}}\right), \forall h \in \Omega_{H}, \forall b \in \Omega_{B} \\
& \sigma_{m, t, h}=\sigma_{m, t-1, h}+\tau\left(\eta_{h}^{c} P_{m, t, h}^{c}-\frac{P_{m, t, h}^{d}}{\eta_{h}^{d}}\right), \forall h \in \Omega_{H}, \forall t \in \Omega_{T}^{b} \backslash\left\{t_{b}^{1}\right\}, \forall b \in \Omega_{B} \\
& 0 \leq \sigma_{m, t, h} \leq E_{h}^{0}+\bar{S}_{S_{m, t, h}^{I}}^{I} S_{h}^{r}, \forall h \in \Omega_{H}, t \in \Omega_{t}^{b}, \forall b \in \Omega_{B} \\
& \widetilde{S}_{m, h}= \begin{cases}\widetilde{S}_{m, h^{\prime}}^{I} & h \in \Omega_{H}^{T} \\
0, & h \in \Omega_{H} \backslash \Omega_{H}^{T}\end{cases} \\
& P_{m, t, h}^{c} \leq \widetilde{S}_{m, h} \bar{P}_{h}^{c}+\bar{P}_{h}^{c, 0}, h \in \Omega_{H}, t \in \Omega_{t}^{b}, \forall b \in \Omega_{B} \\
& P_{m, t, h}^{d} \leq \widetilde{S}_{m, h} \bar{P}_{h}^{d}+\bar{P}_{h}^{d, 0}, h \in \Omega_{H}, t \in \Omega_{t}^{b}, \forall b \in \Omega_{B}
\end{aligned}
$$

The objective Equation (1) minimizes the system costs $V_{m}$ at scenario node $m$ and the variables $\alpha_{j}^{m}$ representing the probability-weighted approximation of future costs across all nodes emanating from $m$. The cumulative discount factors depend on the interest rate $r$ and the considered stage. In particular, the cumulative discount factors for storage investments depend on the unit lifetime $\delta_{h}$ as follows (Appendix A in [32])

$$
r_{h, \epsilon(m)}^{S}=\sum_{i=y_{\epsilon(m)}}^{\min \left\{y_{\epsilon(m)}+\delta_{h}-1, N_{E}\right\}} \frac{1}{(1+r)^{i-1}}
$$

where $N_{E}$ denotes the number of years in the planning horizon.

The dependence on decision variables $x_{m}$ is omitted in Equations (2)-(40) for readability. Investment costs comprise the cost of conventional line reinforcements and storage investments as defined in Equation (3), while system operation costs include generation costs as well as demand curtailment costs across all periods $t$ and demand blocks $b$ as defined in (4). Constraints (5)-(20) relate to investment, (21)-(46) model system operation, while (47) and (48) shown below, describe the future cost function approximation using Benders cuts. Constraint (5) defines the binary nature of conventional investment decisions and, as explained above, is relaxed in all subproblems $\mathbb{P}_{m}^{S}$. The temporal evolution of investments and storage assets decommissioning is described by Equations (6)-(20), where, specifically, (11) limits the number of reinforcements of lines across each scenario, (13) decides the amount of capacity to be built, and (17) limits the size of aggregate energy storage investments. Constraints (6)-(10) couple the investment state variables of the current problem in $m$ with those of the parent node $p(m)$, except for the root node in which case $y_{p(1)}^{I}=0$.

In particular, the available storage capacity (18) at scenario node $m$ is expressed as sum of three terms. The first term accounts for the storage capacity of each technology $h$ built at the previous stages that is available at stage $\epsilon(m)$ considering its building delay. The second terms accounts for the storage decommissioning. It is modelled accounting for the 
built storage capacity at the previous stage considering the delay $\gamma_{h}+\delta_{h}$ that corresponds to the decommissioning time. The last term corresponds to storage capacity built at the current stage and the coefficient assumes values of one only if the specific technology is immediately available.

Note that the duals $\lambda^{I}$ of the complicating variables in these constraints are obtained only in the relaxed subproblems $\mathbb{P}_{m}^{S}$. Finally, $A^{I}, B^{I}$ and $B^{I, 0}$ are auxiliary matrices carrying information of investment delays in a form that enables the transformation of statedependent equations for the application of the temporal decomposition.

In terms of system operation, firstly Constraints (21)-(28) apply to generation assets. The limits on conventional and non-hydro renewable generators are defined by (21), while the ramp rates of all generators are constrained by (22). Constraints (23)-(28) model hydro reservoir and hydro run of river generation. Note that the values $t_{b}^{1}$ and $T_{b}$ denote the first and final time period $t$ in demand block $b$, while $t_{b}^{0}$ is the period immediately preceeding $t_{b}^{1}$ and is used for initialization purposes. Power flow on AC lines is described by the Direct Current Power Flow (DCPF) approximation in (29), while the power flow limits on all lines are imposed with (30). The DCPF is a widely used approximated model in long-term transmission planning problems since it represents a good compromise between accuracy and simplicity. Notice that power losses are ignored which can be a valid assumption in a high voltage transmission system. System balance is enforced with Constraint (31), where the power injections in all buses must equal local demand, considering load curtailment as well. Finally, Equations (32)-(38) model energy storage operation. Specifically, (32)-(34) initialize and update the state of charge of all units in each time period, (35) defines the limits on state of charge, constrained above by the energy capacity, while (37) and (38) limit energy storage charging and discharging power, respectively.

Lastly, in order to approximate the future cost function, Benders cuts are appended to all problems except those pertaining to the final stage of the scenario tree and the master problems in the first iteration $\left(\mathbb{P}_{m}^{M(1)}\right)$. The cuts are generated using the dual variables $\lambda^{I}$ obtained in Constraints (6)-(10) of the relaxed subproblems $\mathbb{P}_{m}^{S}$. Denoting the optimal value of $\mathbb{P}_{m}^{S}$ at iteration $k$ with $\omega_{m}^{S(k)}$, the Benders cuts to be appended to problems $\mathbb{P}_{m}^{S(k)}$ are constructed as

$$
\begin{aligned}
& \alpha_{j}^{m} \geq \omega_{j}^{S(k)}+\sum_{\forall h \in \Omega_{H}^{T}}\left[\sum_{i=1}^{\gamma_{\max }^{S}(h)} \lambda_{j, h, i}^{S(k)}\left(y_{m, h, i}^{S}-y_{m, h, i}^{S(k)}\right)+\sum_{i=1}^{\gamma_{\max }^{S^{R}}(h)} \lambda_{j, h, i}^{S^{R}(k)}\left(y_{m, h, i}^{S^{R}}-y_{m, h, i}^{S^{R}(k)}\right)\right]+\sum_{\forall l \in \Omega_{L}} \sum_{\forall \omega \in \Omega_{W}}\left[\lambda_{j, l, \omega}^{\beta(k)}\left(y_{m, l, \omega}^{\beta}-y_{m, l, \omega}^{\beta(k)}\right)+\right. \\
& \left.\lambda_{j, l, \omega}^{c(k)}\left(y_{m, l, \omega}^{c}-y_{m, l, \omega}^{c(k)}\right)+\sum_{i=1}^{\gamma_{m a x}^{L}(\omega)} \lambda_{j, l, i, \omega}^{F(k)}\left(y_{m, l, i, \omega}^{F}-y_{m, l, i, \omega}^{F(k)}\right)\right], \quad \forall j \in \mathcal{N}^{+}(m), k=1, \ldots, K \\
& \text { while those appended to problems } \mathbb{P}_{m}^{M(k)} \text { as }
\end{aligned}
$$

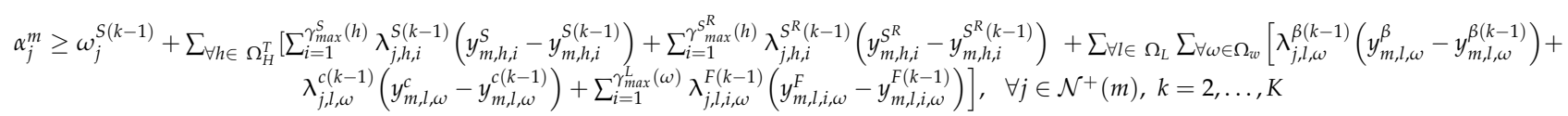

where $K$ denotes a current iteration of the Nested Benders algorithm.

Note that at iteration $k, \mathbb{P}_{m}^{M(k)}$ are solved considering all Benders cuts up to $k-1$, while $\mathbb{P}_{m}^{S(k)}$ include all cuts up to and including $k$.

\subsection{Solution Algorithm and Convergence}

Each iteration of the solution algorithm is made up of two steps (see Figure 1). In the first step, the scenario tree is traversed forward from the root node until the leaf nodes, solving all master problems $\mathbb{P}_{m}^{M}$. In the second step, a backwards pass is performed in the opposite direction solving the dual of the subproblems $\mathbb{P}_{m}^{S}$ in all scenario tree nodes except the root and constructing the Benders cuts. The cuts (39) are appended to subproblems in the same iteration, while cuts (40) are appended to the master problems in the following iteration. The algorithm continues until the bounds become sufficiently close and the lower bound stops improving. The upper and lower bounds in iteration $k$ are described by (41) and (42), respectively, while (43) assesses if the improvement of the lower bound between 
the final two iterations is sufficiently small and (44) checks whether the relative gap is smaller than a chosen value $\epsilon_{g}$.

$$
\begin{gathered}
Z_{u}^{(k)}=\sum_{m \in \Omega_{M}} V_{m}^{M(k)} \\
Z_{l}^{(k)}=\omega_{1}^{M(k)} \\
\frac{Z_{l}^{(k)}-Z_{l}^{(k-1)}}{Z_{l}^{(k-1)}} \leq \epsilon_{l} \\
\frac{Z_{u}^{(k)}-Z_{l}^{(k)}}{Z_{l}^{(k)}} \leq \epsilon_{g}
\end{gathered}
$$

In the above equations, $V_{m}^{M(k)}$ is the optimal cost of the master problem $\mathbb{P}_{m}^{M(k)}$ in the current iteration $k$. The described algorithm is illustrated in Figure 1.

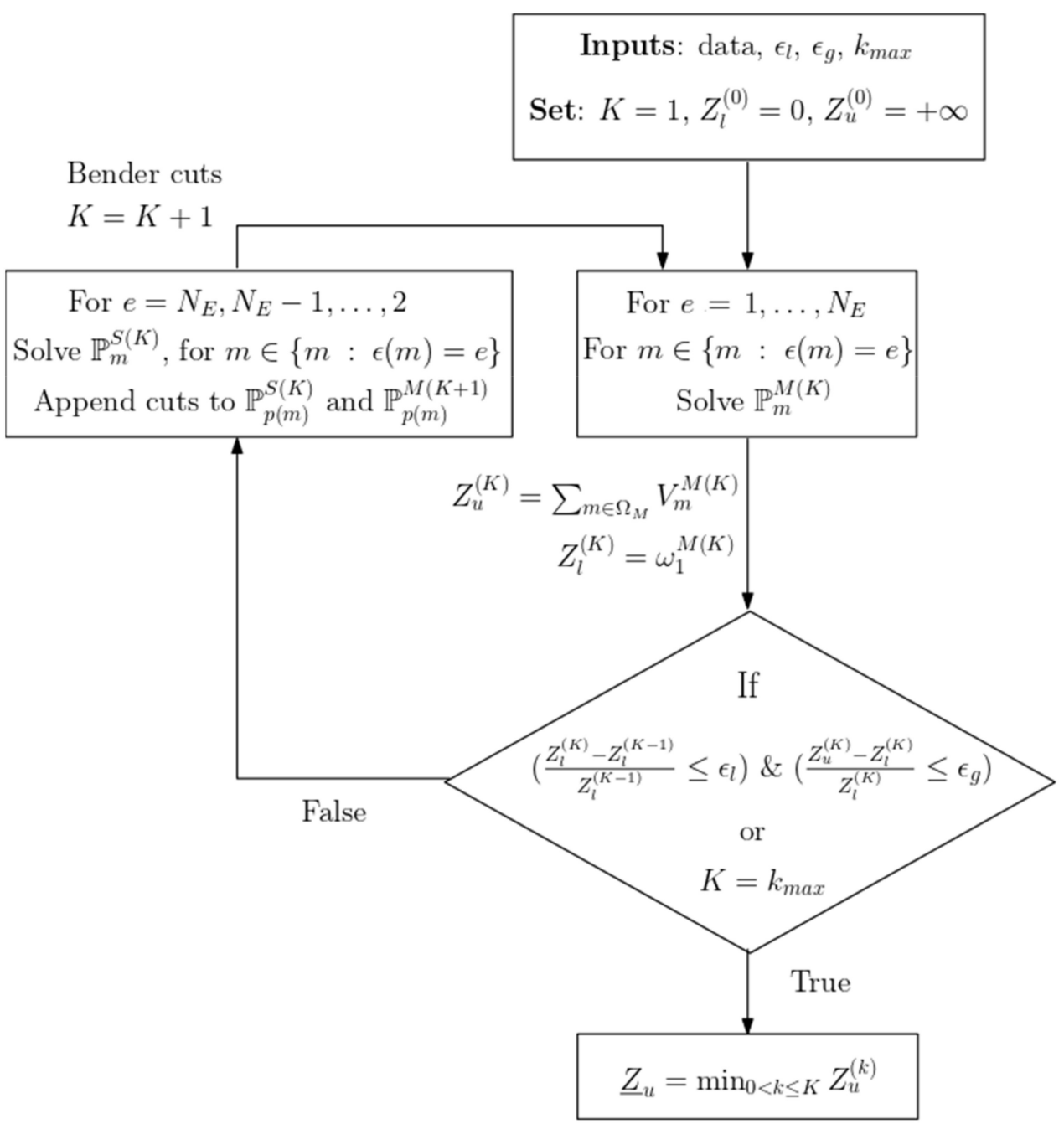

Figure 1. Diagram of the decomposition algorithm.

The convergence criterion assessment is always performed after the completion of step 1 of the algorithm. The algorithm returns a lower and an upper bound to the optimal cost of the original problem. The solution with the smallest upper bound $\underline{Z}_{u}$ is the best approximation of the optimal solution, and its degree of optimality is certified by the 
lower bound $Z_{l}^{(k)}$. Should the above criterion not be satisfied after a pre-defined maximum number of iterations, the approximation of the lower bound could be additionally improved by convexification schemes, such as those proposed in [32].

\section{Case Study}

\subsection{Description}

The aim of the current study is to analyze the investment needs of India's electricity transmission system until the year 2060, taking into account official data for its electricity demand (including quantities such as peak demand and annual load profiles for all different states of the country) and its generation (including quantities such as renewable and thermal installed capacity as well as high-resolution solar and wind profiles). The system planner is in year 2020 and is solving a stochastic optimization problem that will yield the location, amount and type of investments required with focus on years 2020, 2030, 2040 and 2050. In addition, uncertainty has been considered to significantly affect the future evolution of the investment cost of battery storage, the installed capacity of solar PV units and the installed capacity of wind units. Particular focus has been placed on the battery storage technology and its role for India's ongoing transition to greater penetration of renewable in the system. A description of the India's electricity system follows as well as of the electricity transmission system in general.

India's electricity transmission grid consists of five regions. These include the Northern Region (NR), Southern Region (SR), Western Region (WR), Eastern Region (ER), and North-Eastern Region (NER), as shown in Figure 2 below, and each is operated by a regional load dispatch center that coordinates with state load dispatch centers located in different states of the corresponding region.

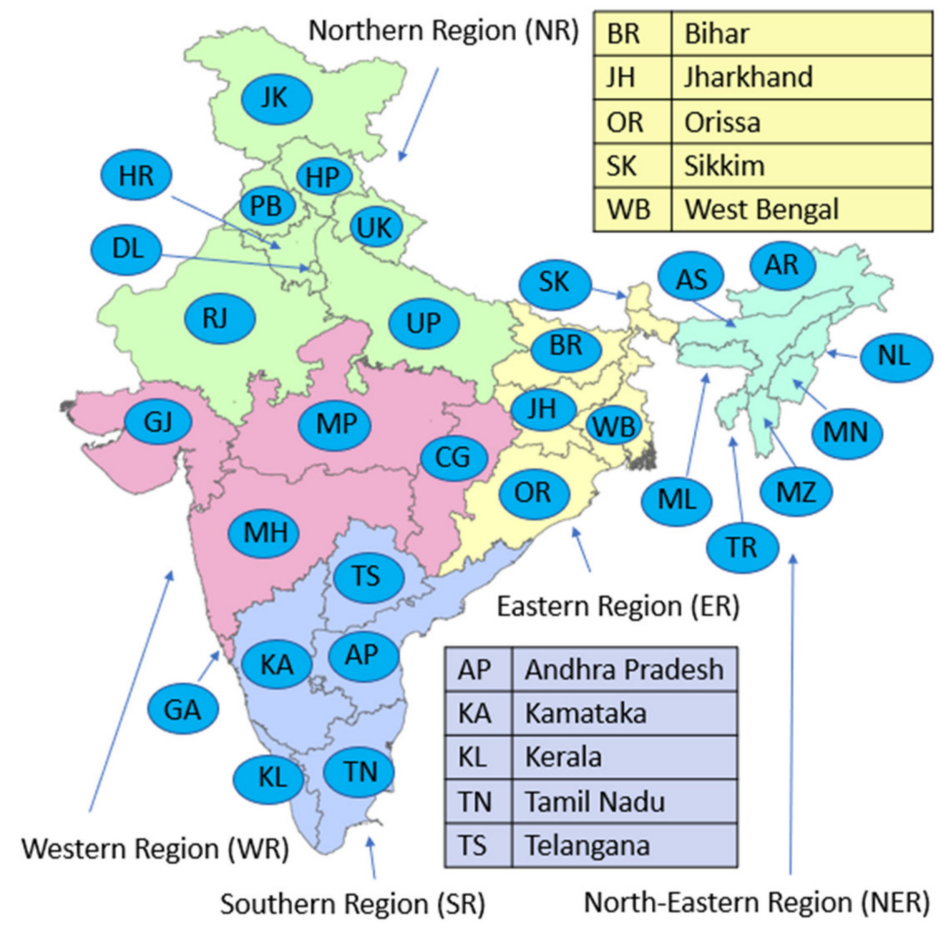

\begin{tabular}{|l|l|}
\hline AR & Arunachal Pradesh \\
\hline AS & Assam \\
\hline ML & Meghalaya \\
\hline MN & Manipur \\
\hline MZ & Mizoram \\
\hline NL & Nagaland \\
\hline TR & Tripura \\
\hline CG & Chhattishgarh \\
\hline GA & Goa \\
\hline GJ & Gujarat \\
\hline MH & Maharashtra \\
\hline MP & Madhya Pradesh \\
\hline DL & NCT of Delhi \\
\hline HP & Himachal Pradesh \\
\hline HR & Haryana \\
\hline JK & Jammu and Kashmir \\
\hline PB & Punjab \\
\hline RJ & Rajasthan \\
\hline UK & Uttarakhand \\
\hline UP & Uttar Pradesh \\
\hline
\end{tabular}

Figure 2. Diagram of the electricity grid of India, which consists of 5 regions and 30 states (shown as blue circles along with their abbreviations).

In the current model, a total of 30 busbars are considered, which represent 29 states and one Union Territory (UT)-NCT of Delhi. The reason behind considering this UT as a separate bus is its high electricity demand and low opportunity for installation of generation units. In addition, each of the 30 states/UT hosts 11 different generators (each of a different type); that is $30 \times 11=330$ generators/storage units are assumed in the model. 
The 11 generator types are as follows: gas CCGT, coal, hydro reservoir, hydro run of river, pumped hydro storage, nuclear, oil, gas OCGT, biomass, solar PV, and wind. Notice that the model does not invest in electricity generation, which is considered to be an input parameter. Note that for the maximum potential of these units on a state-by-state basis official data have been used. Data related to capacity factors, as well as availability of solar $\mathrm{PV}$ and wind units, are referred from [1].

A total of 62 transmission lines were considered, six of which are HVDC and the rest are HVAC as can be seen in Figure 3. For each of the lines, official data for their thermal capacity (MW) as well as their length $(\mathrm{km})$ and line reactance were included in the model.

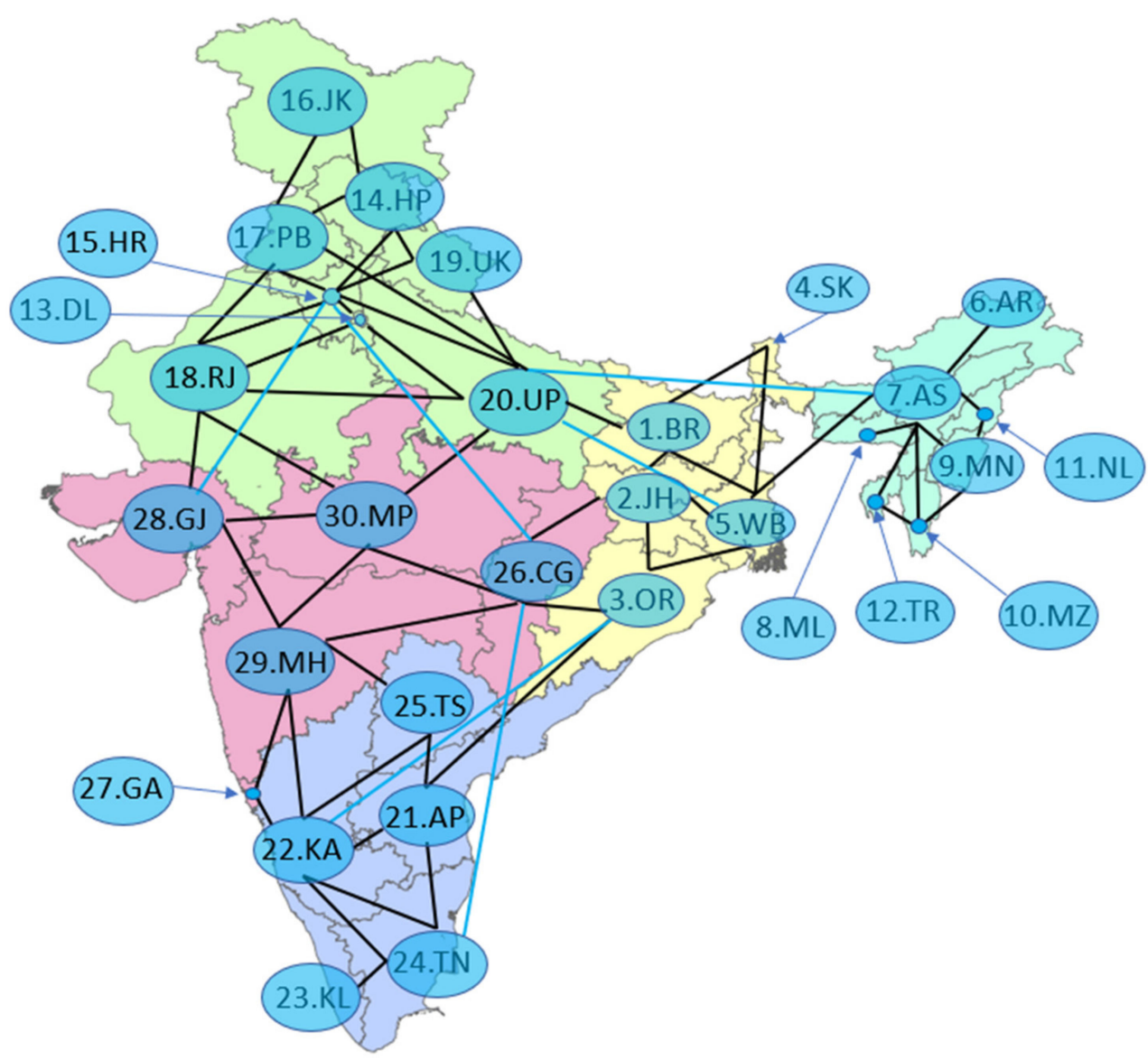

Figure 3. Diagram of the electricity grid of India, with the 62 transmission lines appearing where six of them are HVDC (shown in blue).

As mentioned, the presence of multiple sources of uncertainty has a significant effect on system operation and investment. Specifically, uncertainty is represented via a scenario tree, shown in Figure 4, consisting of 11 scenario-tree nodes, 4 scenarios and 4 epochs/stages. The first scenario (S1) consists of nodes 1, 2, 4 and 8. Scenario 2 consists of nodes 1, 2, 5, and9. Then, scenarios 3 and 4 consist of nodes 1, 3, 6, and 10 and 1, 3, 7, and 11, respectively. In addition, the first epoch consists of node 1, while the second epoch consists of nodes 2 and 3 . Then, the third epoch consists of nodes $4-7$ and the fourth epoch consists of nodes 8-11. In addition, epoch1 spans the period from 1 January 2020 until 31 December 2029. Epoch2 goes from 1 January 2030 until 31 December 2039, while epoch3 goes from 1 January 2040 until 31 December 2049. Finally, the fourth epoch covers the period from 1 January 2050 until 31 December 2059. Notice that the scenario tree displays the probabilities of transition from one node (state of the system) to another. 


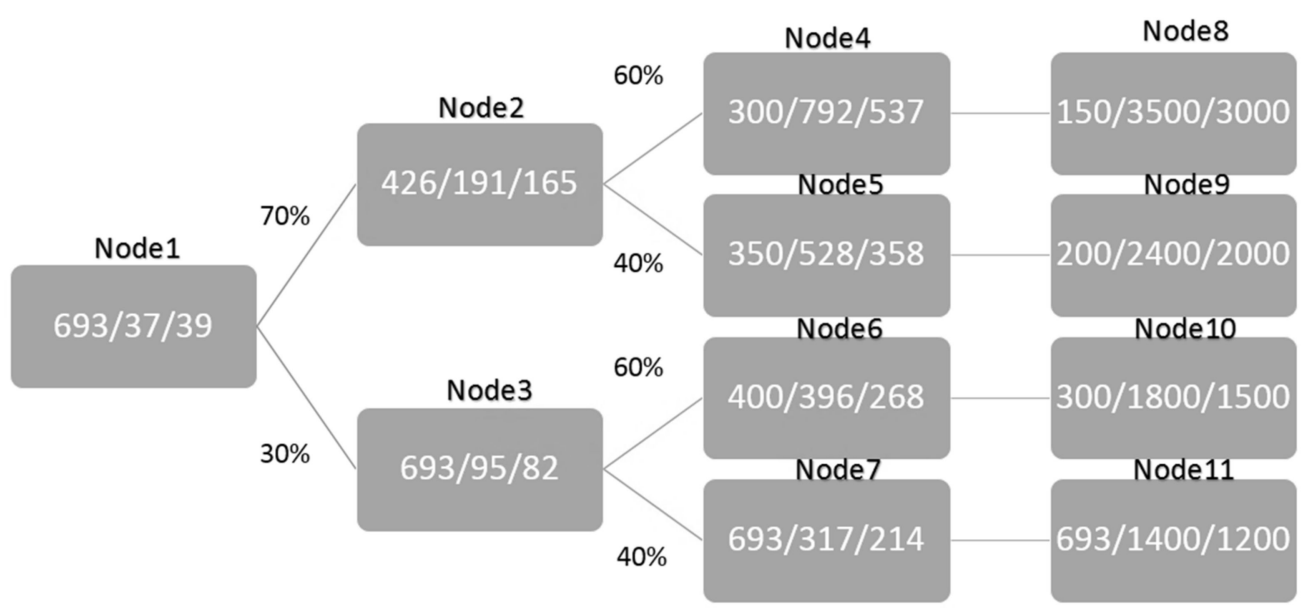

Figure 4. Scenario tree structure, consisting of 11 scenario-tree nodes, illustrating the uncertainty around three quantities in the following order; namely around battery storage investment cost (in $£ \mathrm{k} / \mathrm{kW}$ ), around the installed generation capacity of solar PV across India (GW) and around the installed generation capacity of wind units across India (GW). Note that epoch1 (1 January 2020-31 December 2029) covers node 1, and epoch2 (1 January 2030-31 December 2039) covers nodes 2-3, while epoch3 (1 January 2040-31 December 2049) covers nodes 4-7 and epoch4 (1 January 2050-31 December 2059) covers nodes 8-11. Very high values for wind and solar were applied to the period 2050-2060 (last epoch) to also account for future increases beyond the problem horizon. The values over the straight lines connecting nodes indicate probabilities of transition (e.g., it is $60 \%$ likely to go from node 3 to node 6), while the probability from nodes of the third epoch (nodes 4-7) to those of the last epoch (nodes $8-11$ ) is $100 \%$ (i.e., deterministic transition).

In this context, demand is expected to increase over the coming decades in all India states [33] as shown in Figure 5 below, where this demand is assumed to take place in a deterministic fashion. Therefore, given the increase in demand as well as in generation capacity, particularly that of renewables, the network planner may need to make significant amounts of investment in the grid. The available technologies for investment include both energy storage and conventionally upgrading existing transmission lines. One key difference between these two technologies is the "build-time", which is the amount of time (measured in epochs) required for the investment to become operational. In this context, it takes one epoch for the conventional investment to become operational (e.g., if it is decided to upgrade one line in epoch1, then the line will become operational in epoch2), while for energy storage the build time is equal to zero epochs. This difference reflects the fact that typically conventional investments involve lengthy licensing procedures as well as complex mechanical works that may cause delays. Hence, conventional investments are particularly faced with the prospect of the risk of stranded assets, where under uncertainty a conventional investment may end up having low utilization rates due to an unfavorable realization of uncertainty.

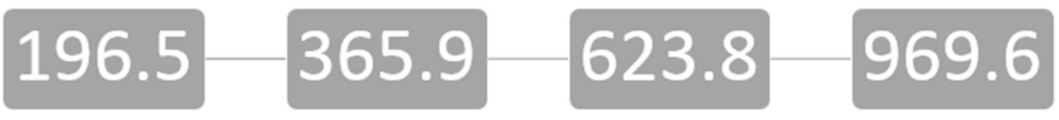

Figure 5. Peak demand (GW) across India for each of the four epochs (2020-2029, 2030-2039, 2040-2049, 2050-2059).

In terms of cost, investing in a line involves a variable investment cost equal to GBP 100/km/MW/year and a fixed investment cost of GBP 200,000/km/year and GBP $400,000 / \mathrm{km} /$ year for HVAC and HVDC lines, respectively. In addition, a $10 \%$ discount rate, which is typical for India, and a 25-year lifetime for line reinforcements as well as a 20-year lifetime for battery storage were used in the model [34]. A high-efficiency battery 
storage technology was assumed with a four-hour duration (i.e., it takes $4 \mathrm{~h}$ to fully charge from zero, or to discharge from full capacity) and with its investment costs exhibiting uncertainty. Notice that the cost stays the same across the fourth scenario, while it reduces at the fastest rate across scenario 1.

The subsection that follows presents the results of the study.

\subsection{Results}

Given that the electricity demand is expected to increase over the next decades, as well as the installed capacity of many generators, and mainly renewables, the network planner needs to invest in the electricity transmission system in order for future power flows to be safely accommodated. The investment decisions are taken in the years 2020 (first year of epoch1), 2030 (first year of epoch2), 2040 (first year of epoch3) and 2050 (first year of epoch4) and there are two technologies that are available to the planner for investment: upgrade of the capacity (MW) of one or more transmission lines (known as the "conventional network reinforcement") and battery energy storage. Given that there are two technologies, the case study has been performed twice; namely with and without the possibility to invest in energy storage, while in both cases conventional reinforcements are available to the planner. Table 1 summarizes the costs associated with both expansion strategies as well as shows the obtained Option Value of energy storage.

Table 1. Expected costs and Option Value of energy storage.

\begin{tabular}{ccc}
\hline & Conventional Reinforcement Only & $\begin{array}{c}\text { Conventional Reinforcement and } \\
\text { Storage Investment }\end{array}$ \\
\hline Expected investment cost (GBP) & $12,097,947,208$ & $26,732,242,295$ \\
Conventional reinforcement (GBP) & $12,097,947,208$ & $10,123,219,872.03$ \\
Energy storage (GBP) & $/$ & $16,609,022,423$ \\
Expected system operation cost (GBP) & $507,853,578,872$ & $480,318,047,587$ \\
Expected total system cost (GBP) & $519,951,526,080$ & $507,050,289,882$ \\
Option Value (GBP) & $/$ & $12,901,236,198$ \\
\hline
\end{tabular}

The results presented in the above table confirm the significant economic benefit of including energy storage as an investment option for network expansion. The Option Value of energy storage obtained in the context of the long-term expansion of the transmission network in India is more than GBP 12.9 bn and it is calculated as the difference between the expected total system cost with and without energy storage. In other words, it represents the net economic benefit, under uncertainty, accrued when energy storage is deployed in the system. The primary driver of this value is the substantial savings in system operation that energy storage enables through its operational flexibility. Specifically, system operation savings amount to GBP $27.5 \mathrm{bn}$. In addition, the expected cost of conventional network reinforcements is reduced by around GBP 1.98 bn, which demonstrates the value of strategic flexibility of investing in energy storage.

The optimal investment strategy for both cases, in terms of location and type of investments, is presented in Figures 6 and 7, where (i-j) represent line capacity upgrades between buses $\mathrm{i}$ and $\mathrm{j}$, and ( $\mathrm{z}$ ) represent energy storage investments in bus (i.e., India state) $\mathrm{z}$.

\subsection{Discussion}

\subsubsection{Investment Flexibility}

Some important observations can be made based on the results presented in the previous subsection. Firstly, the differences between Figures 6 and 7 demonstrate the ability of energy storage to defer or displace conventional line reinforcements. Specifically, the possibility to invest in energy storage allows to displace the investment in line (1-4) in the root node. Then, in stage 2 , notice that the conventional investment decisions differ between the two cases as a result of different power flows due to optimal energy storage operation. There is additionally deferral of the investment in line (14-19) from 
node 3 to nodes 6 and 7. Finally, energy storage investments are able to displace a large number of conventional reinforcements in all nodes in stage 3 . These aspects lead to fewer conventional reinforcement projects and a lower cumulative capacity upgrade. Consequently, conventional investment costs are lower in all scenarios and the risk of overinvestment in scenarios with lower demand growth is reduced. Notice also that when storage is not available to the planner (Figure 6), there are no investments taking place in the very last epoch because conventional reinforcement entails a one-epoch build time.

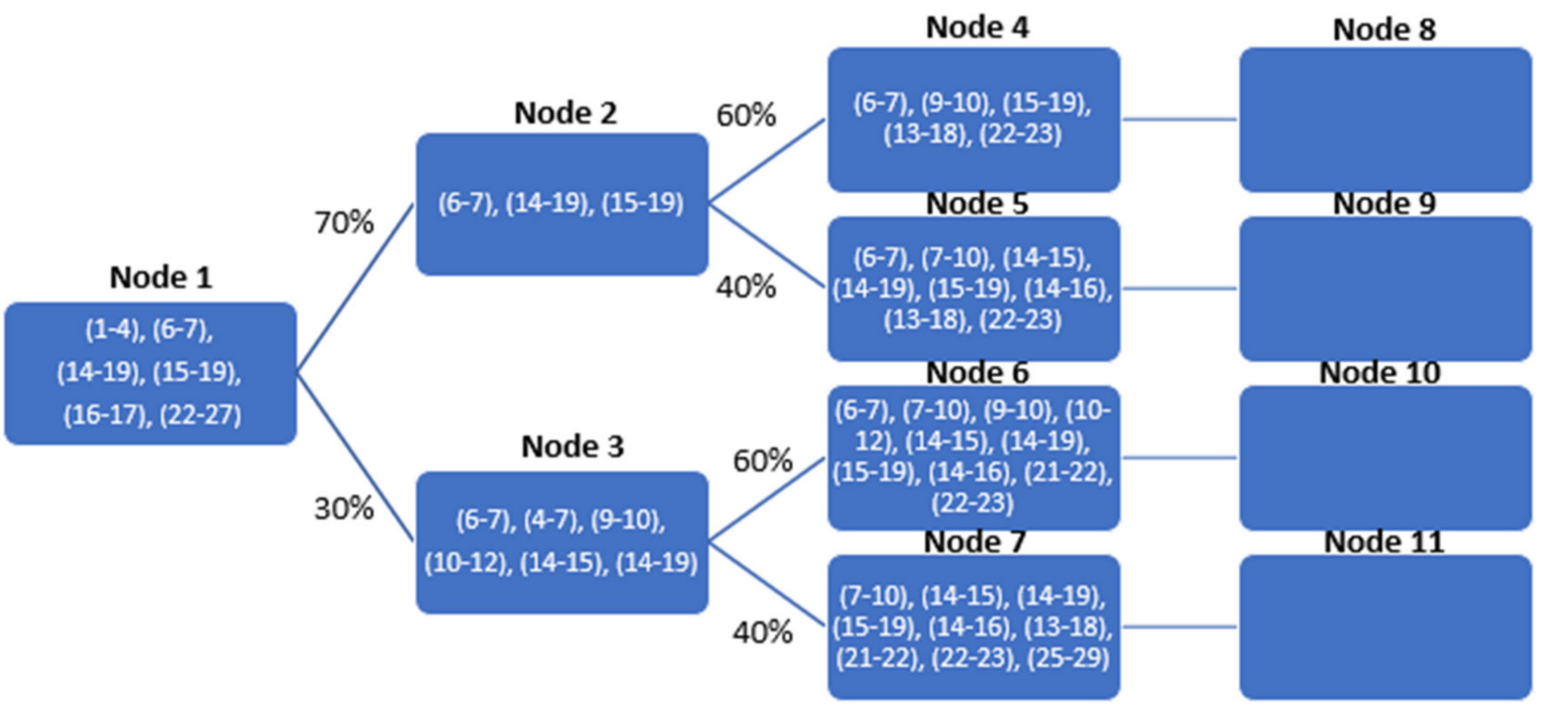

Figure 6. Optimal investment strategy for the case with only conventional investments. The notation (i-j) represents the upgrade of the capacity of the transmission line connected buses (i.e., India states) $i$ and j. Note that the scenario tree depicts investment decisions, i.e., the capacity upgrades will become operational one epoch afterwards (this is why there are no investment decisions taken in the last epoch as there is no epoch5).

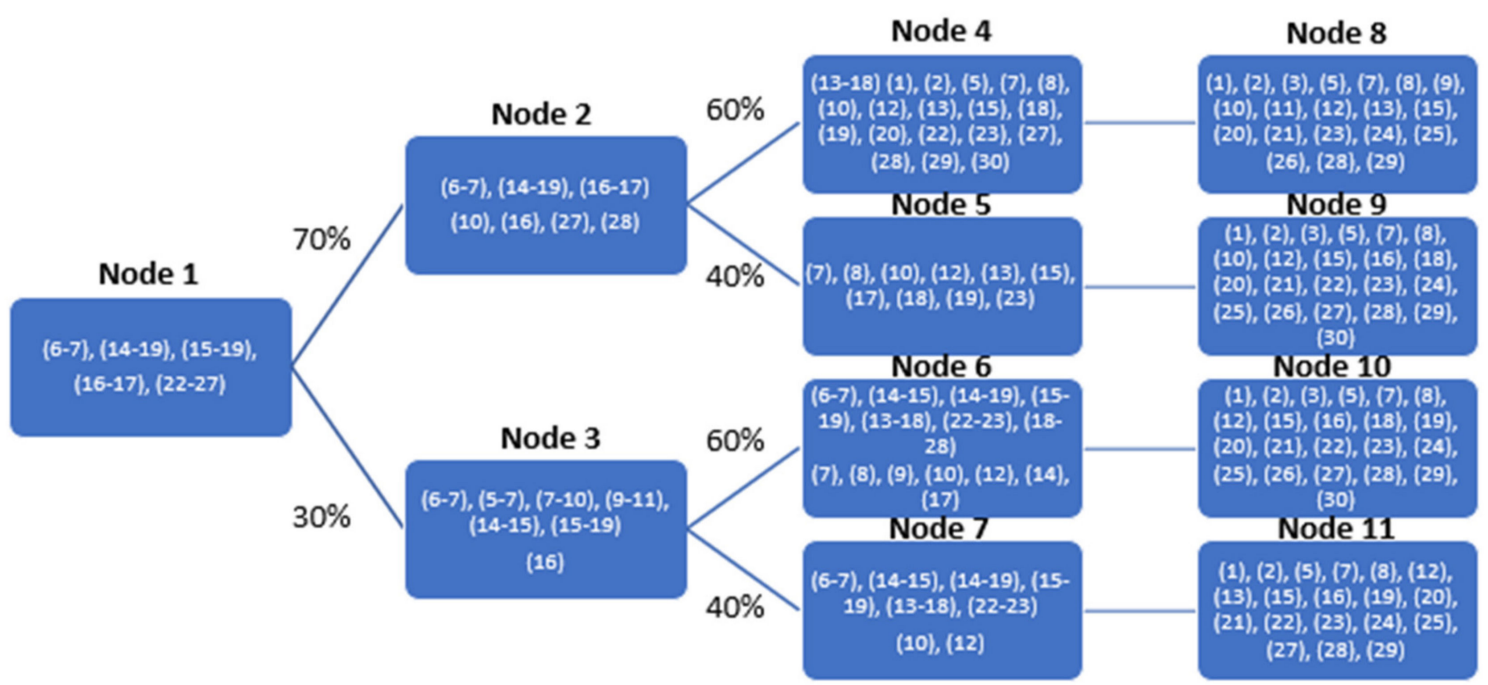

Figure 7. Optimal investment strategy for the case with conventional and energy storage investments. The notation (z) represents energy storage investment in bus (i.e., India state) $\mathrm{z}$.

Figures 8 and 9 show all investments in the transmission system of India until the year 2050 following scenario 1 for both cases-with and without the ability to invest in energy storage. Colored lines represent conventional reinforcements and squares around the network buses represent investment in energy storage in the corresponding state. The colors correspond to the size of investments in terms of capacity, according to the legends of Figures 8 and 9. The figures show that a large number of investments are concentrated 
in the northern part of the country, followed by several reinforcements in the northeast and south. Figure 9 shows that there are fewer lines that need reinforcement and that the capacity investments are lower when energy storage investments are made. Furthermore, the large-scale investments in storage highlight its value in system operation, particularly in facilitating the integration of renewable generation.

\subsubsection{Future Generation Mix}

Another noteworthy comparison is the expected generation mix in 2050, for example between scenario-tree node 8, (year 2050, scenario 1), scenario-tree node 11 (year 2050, scenario 1), and scenario-tree node 1 (year 2020), as shown in Figures 10-12, respectively. Figure 10 involves high capacity of energy storage and high installed capacities for wind and solar PV, while the opposite applies to node 11 (Figure 11), whereas scenario-node 1 involves the smallest capacity of wind, solar and storage. The figures show four typical days of power system operation in each season, where time blocks 1, 2, 3 and 4 correspond to winter, summer, monsoon, and autumn, respectively. It can be observed that while renewables have a limited presence in the mix of year 2020 (Figure 12), this radically changes in year 2050, specifically under scenario-tree node 8 (Figure 10 as compared to Figure 11).

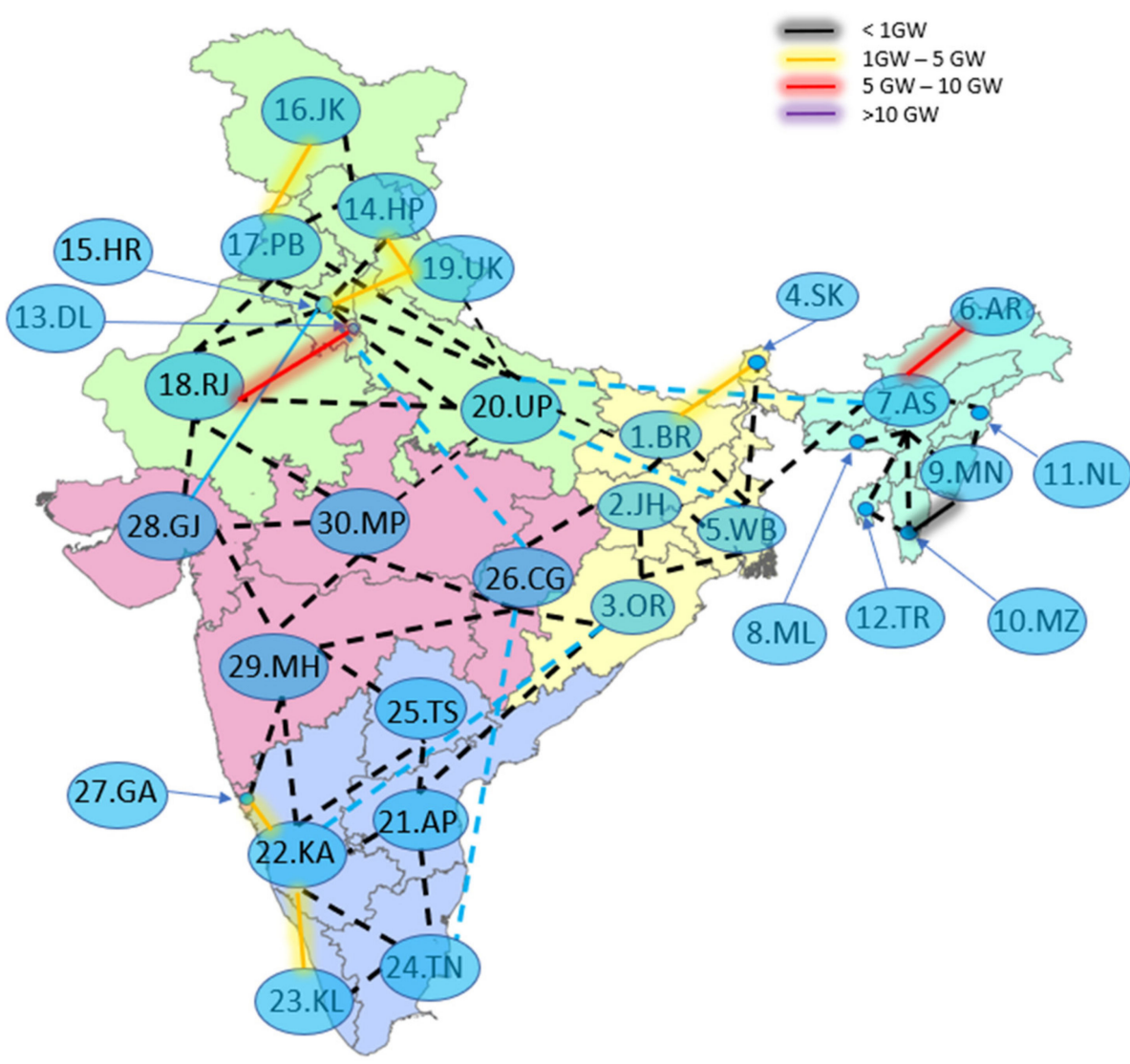

Figure 8. Aggregate investments in the transmission system of India as of 2050 (scenario-tree node 8) with conventional reinforcements only. Dashed lines represent existing transmission lines and full lines represent transmission lines that are reinforced. 


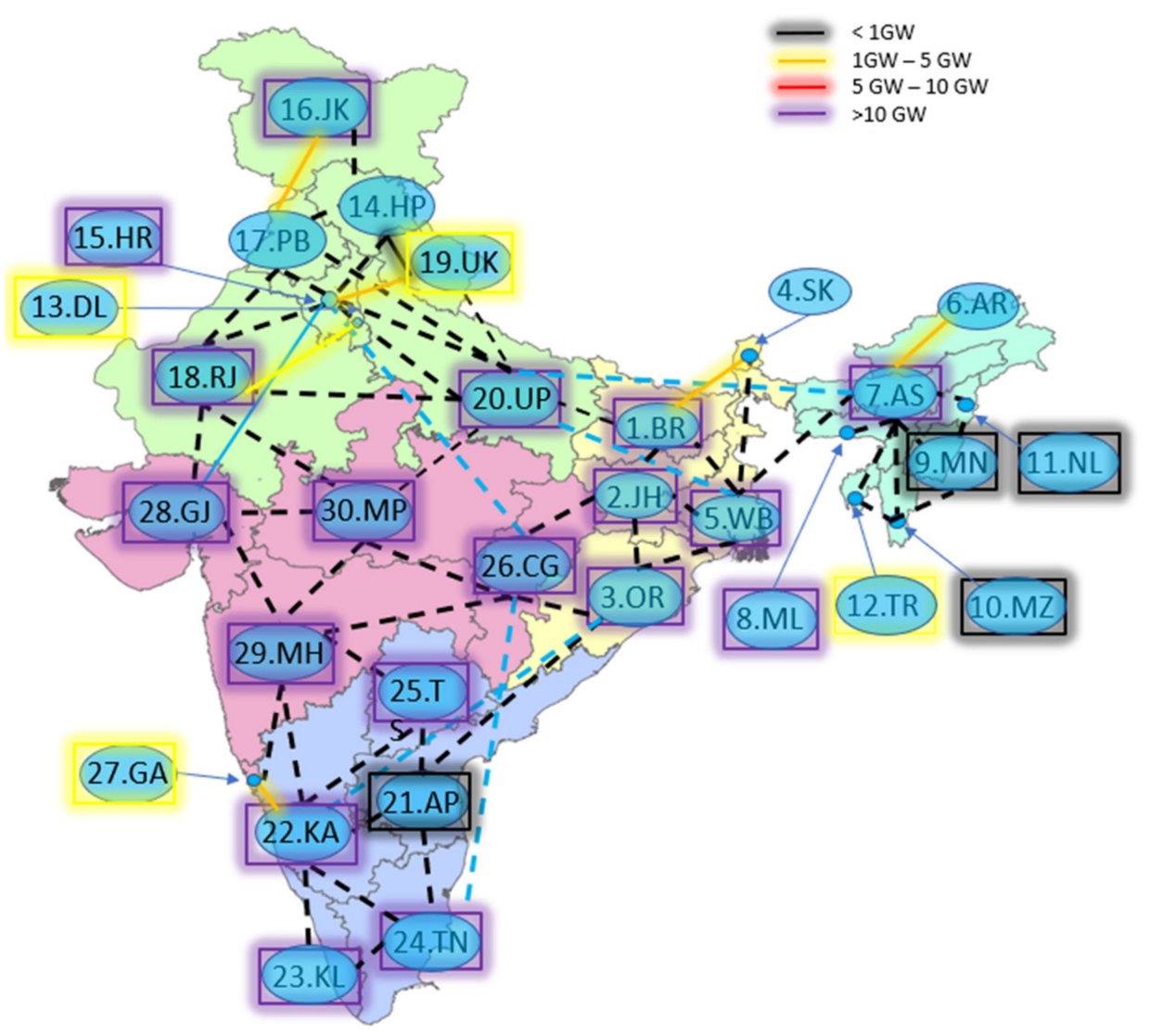

Figure 9. Aggregate investments in the transmission system of India as of 2050 (scenario-tree node 8 ) with the possibility to invest in conventional reinforcements and in energy storage. Dashed lines represent existing transmission lines, full lines represent transmission lines that are reinforced, and squares represent energy storage investment in the corresponding bus.
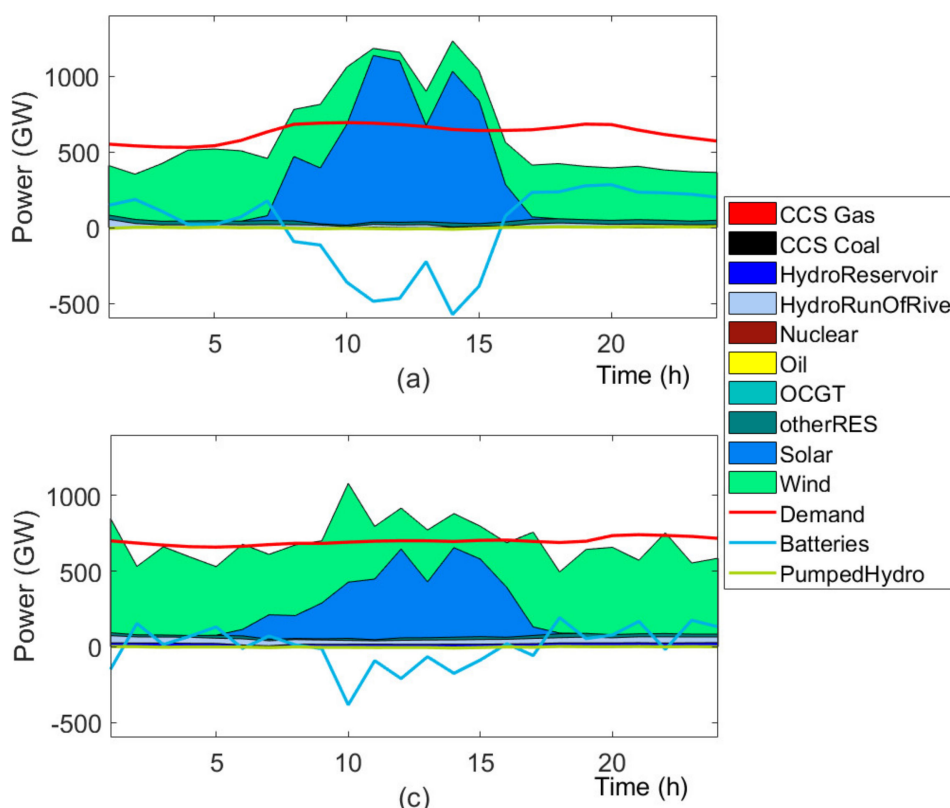

(c)

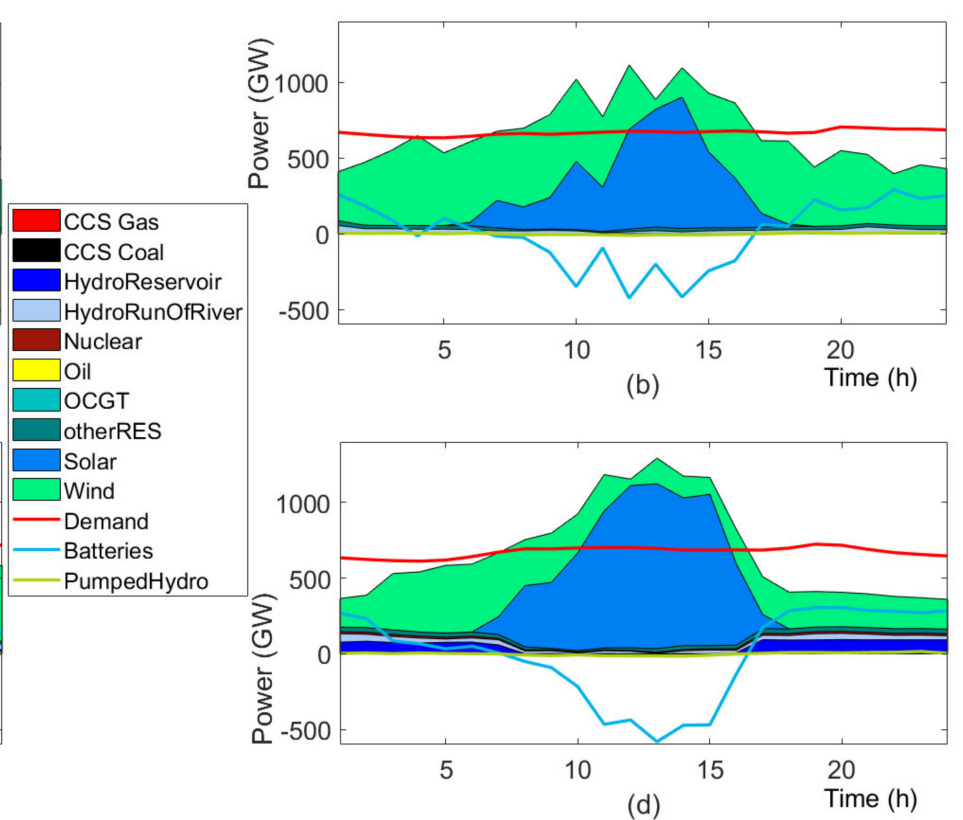

(d)

Figure 10. Generation mix in the year 2050 according to scenario 1 (i.e., scenario-tree node 8), with the availability of storage, in four typical days. Figure (a) reports a typical day within the period January-March, (b) a typical day within April-June, (c) a typical day within July-September, and (d) a typical day within October-December. 

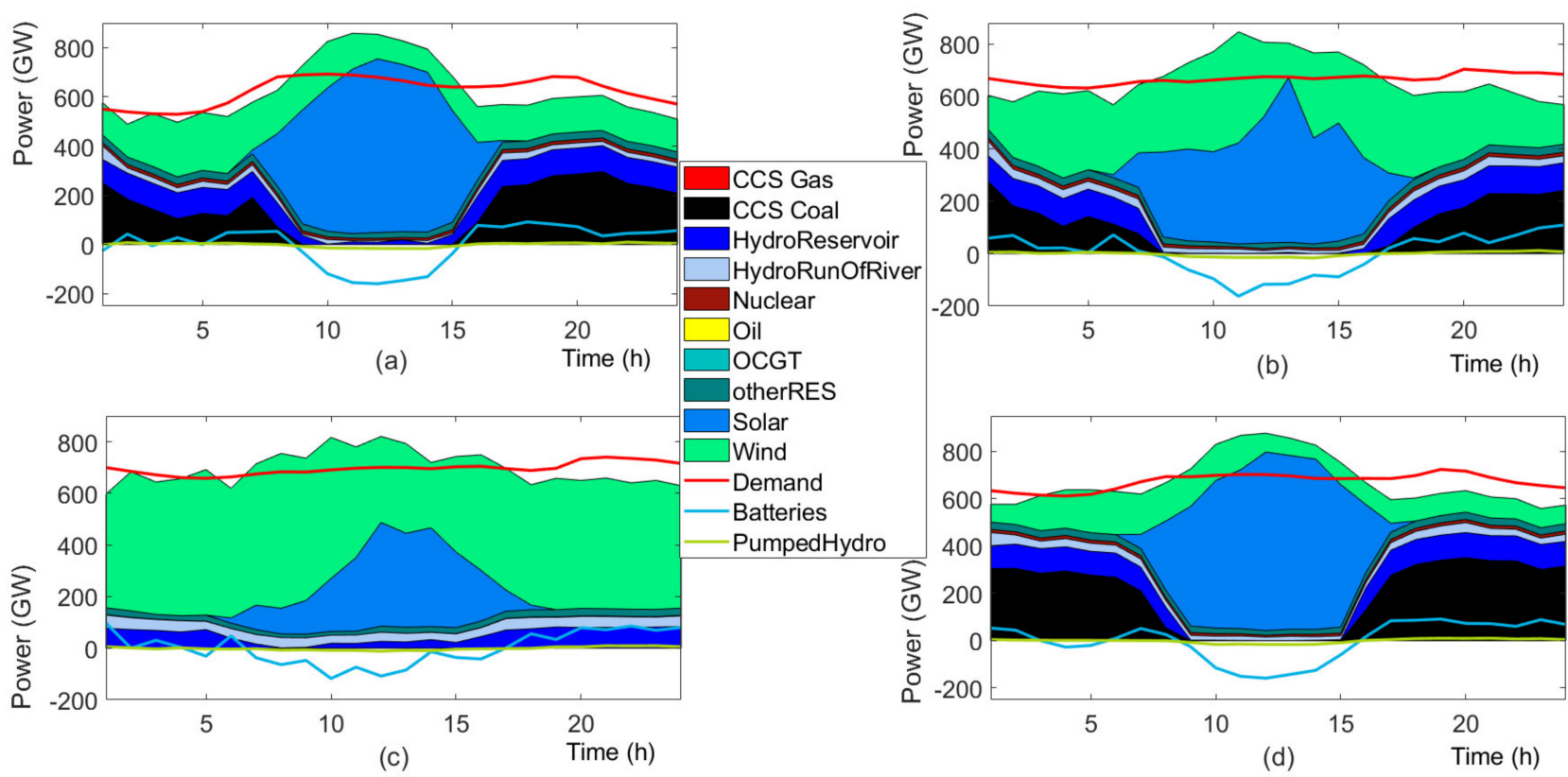

Figure 11. Generation mix in the year 2050 according to scenario 4 (specifically, scenario-tree node 11), with the availability of storage, in four typical days. Figure (a) reports a typical day within the period January-March, (b) a typical day within April-June, (c) a typical day within July-September, and (d) a typical day within October-December.
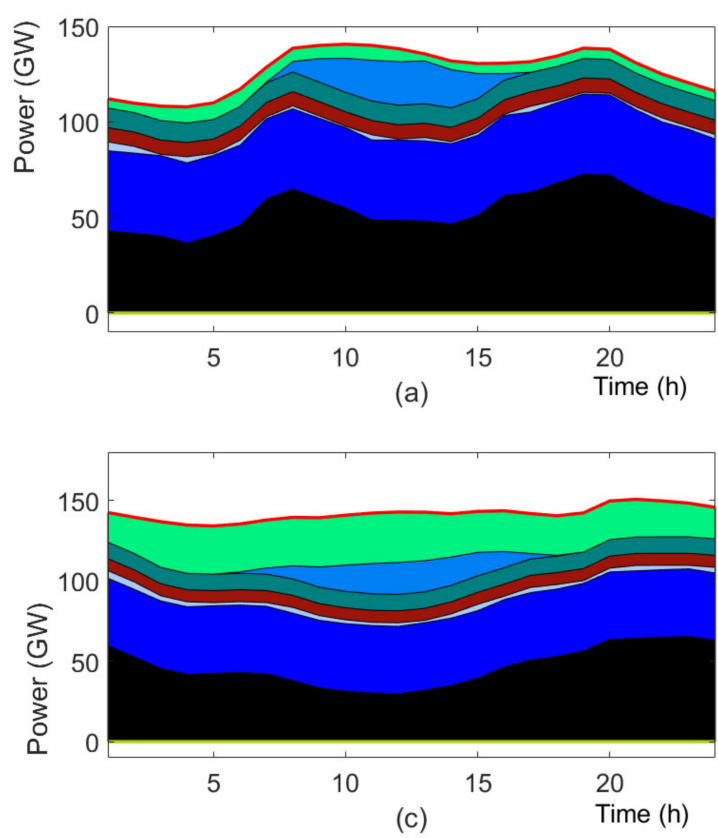
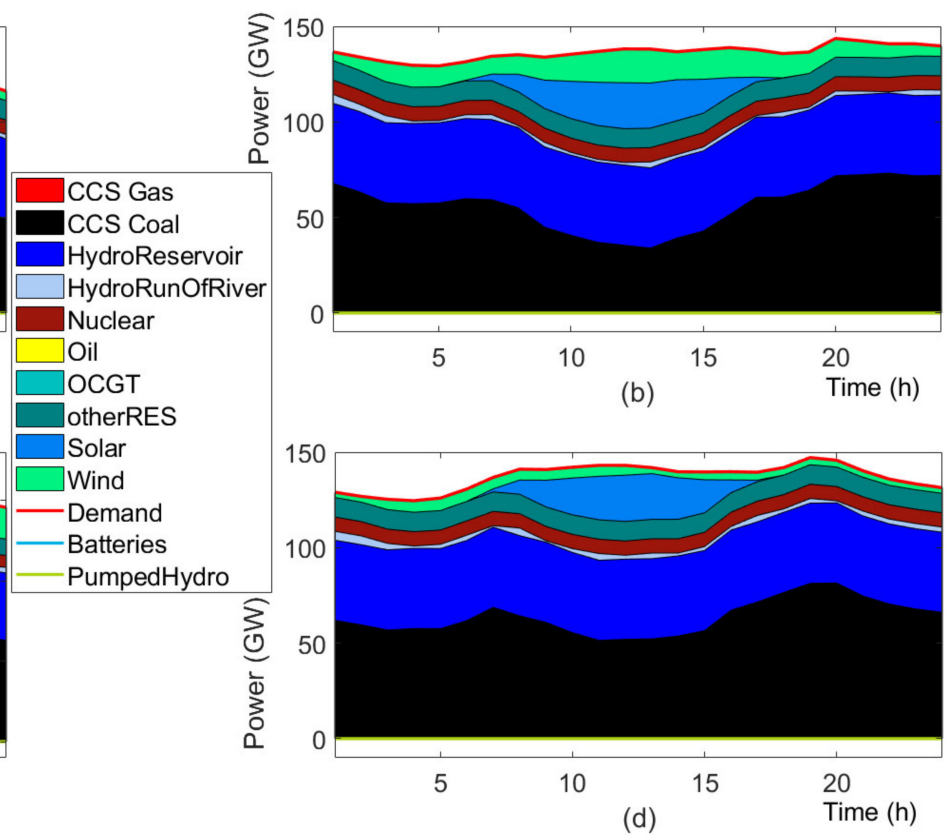

Figure 12. Generation mix in the year 2020 (specifically, scenario-tree node 1), with the availability of storage, in four typical days. Figure (a) reports a typical day within the period January-March, (b) a typical day within April-June, (c) a typical day within July-September, and (d) a typical day within October-December.

In addition, Figures 13 and 14 below show the generation mix in scenario-nodes 8 and 11 , for the study where energy storage is not available to the planner. By contrasting Figure 13 with Figures 10 and 14 with Figure 11 we can observe the role that energy storage plays in helping incorporating renewables into the system, thereby reducing the reliance on fossil fuels. 


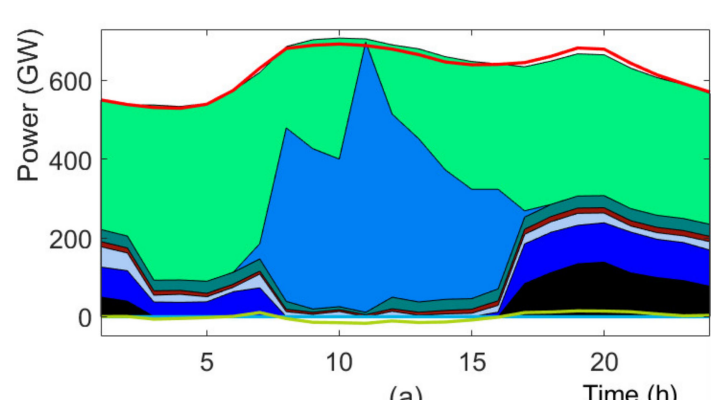

(a)

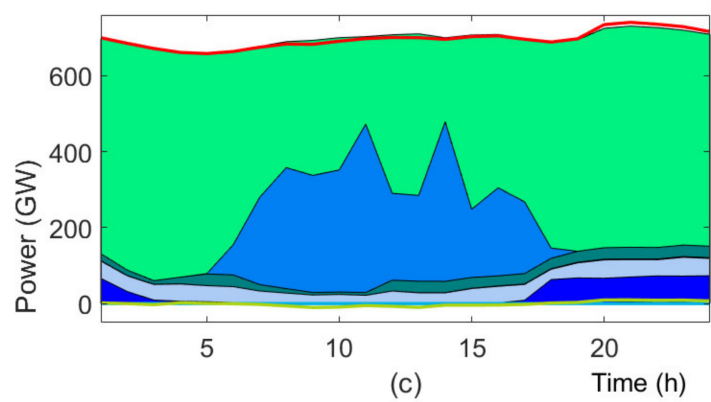

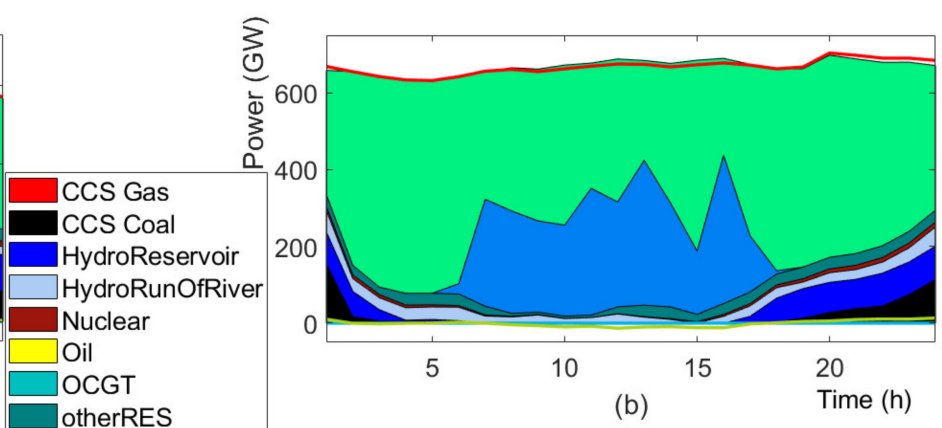

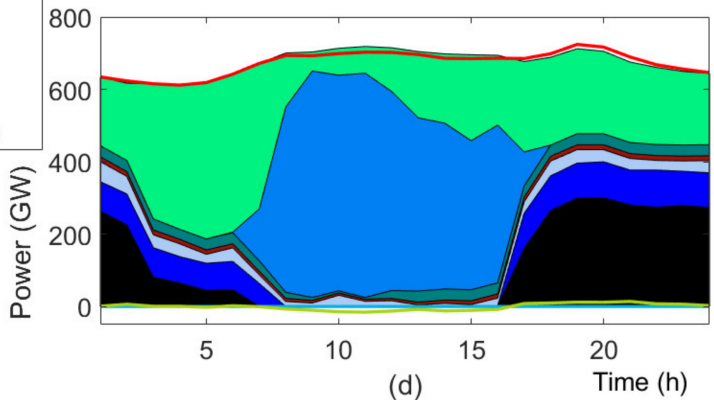

Figure 13. Generation mix in the year 2050 (specifically, scenario-tree node 8), without the availability of storage, in four typical days. Figure (a) reports a typical day within the period January-March, (b) a typical day within April-June, (c) a typical day within July-September, and (d) a typical day within October-December.

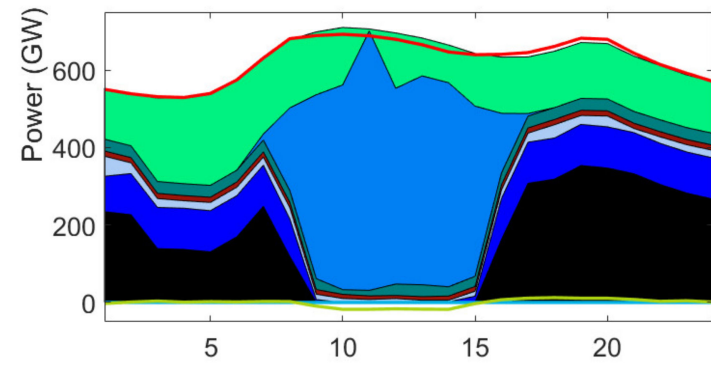

(a)

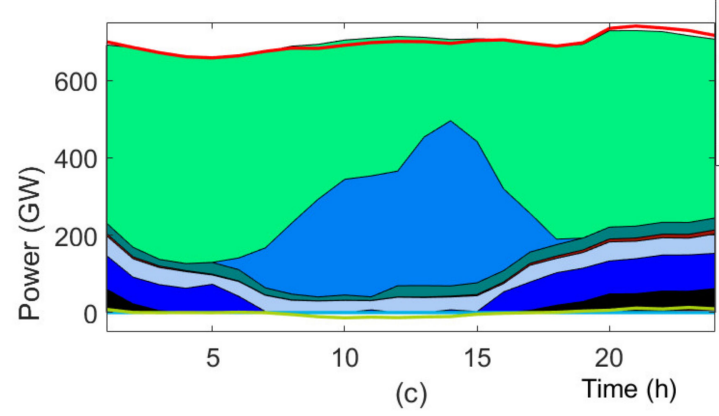

(c)

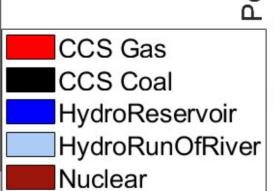

Nuclear

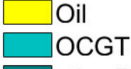

OCtherRES
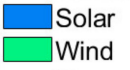

Wind PumpedHydro
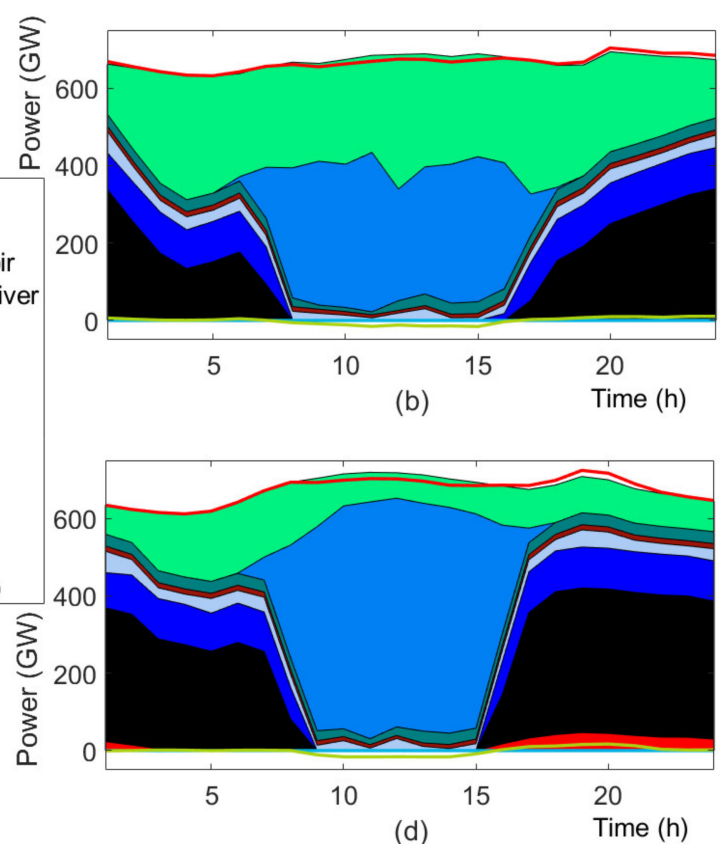

Figure 14. Generation mix in the year 2050 (specifically, scenario-tree node 11), without the availability of storage, in four typical days. Figure (a) reports a typical day within the period January-March, (b) a typical day within April-June, (c) a typical day within July-September, and (d) a typical day within October-December.

Firstly, from the above figures it is evident that the presence and utilization of renewable generation is higher in scenario-tree node 8 since it belongs to scenario 1 which entails high installed generation capacity in solar PV and wind. In addition, scenario-tree node 8 entails large investment in storage (see Figure 9) since scenario-node 8 involves the smallest storage investment cost. Notice that in the plots in Figure 10, the total generation exceeds demand for several hours around midday when solar generation is abundant as the invested storage units are able to charge during this period. In other periods of the day, 
demand is partially supplied with this previously stored energy through the discharging of the storage units, thus effectively managing network congestion and accommodating a larger share of renewable generation. This observation is true for all seasons; nevertheless, the proportions of renewable generation sources changes throughout the year.

Figure 11 shows a different pattern for the typical generation mix in the year 2050 according to scenario 4, i.e., in scenario-tree node 11, characterized by less renewables than scenario node 8 and higher storage investment cost. Unlike the previous case, due to the lower storage installed capacity in this scenario, surplus generation is lower and occurs in various hours throughout the day, while only a smaller proportion of the demand is covered by energy storage units. In addition, renewable generation does not displace coal generation to the same extent as in the previous case.

These observations show that storage operation contributes to lower expected total system costs. Conversely, the absence of energy storage effectively constrains the utilization of renewable generation, especially solar and wind, leading to more carbon-intensive based generation and higher system operation costs. This comparison demonstrates the benefit of integrating energy storage in network expansion planning beyond its ability to offer investment flexibility, i.e., the ability to defer and displace bulky and irreversible conventional reinforcements.

In addition, by looking at the generation mix for each region in scenario-node 8 as reported in Figures 15-19, we can observe the high renewable penetration across all regions. Given the high amount of renewables, energy storage plays a pivotal role in storing this energy and utilizing it so as to reduce the output from non-clean sources of energy. This applies to all regions and explains the significant amount of energy storage investment across India, as witnessed in Figure 9. This figure shows that most storage is placed in the western part of India since as the following figures show there is very high generation from renewables in these regions.
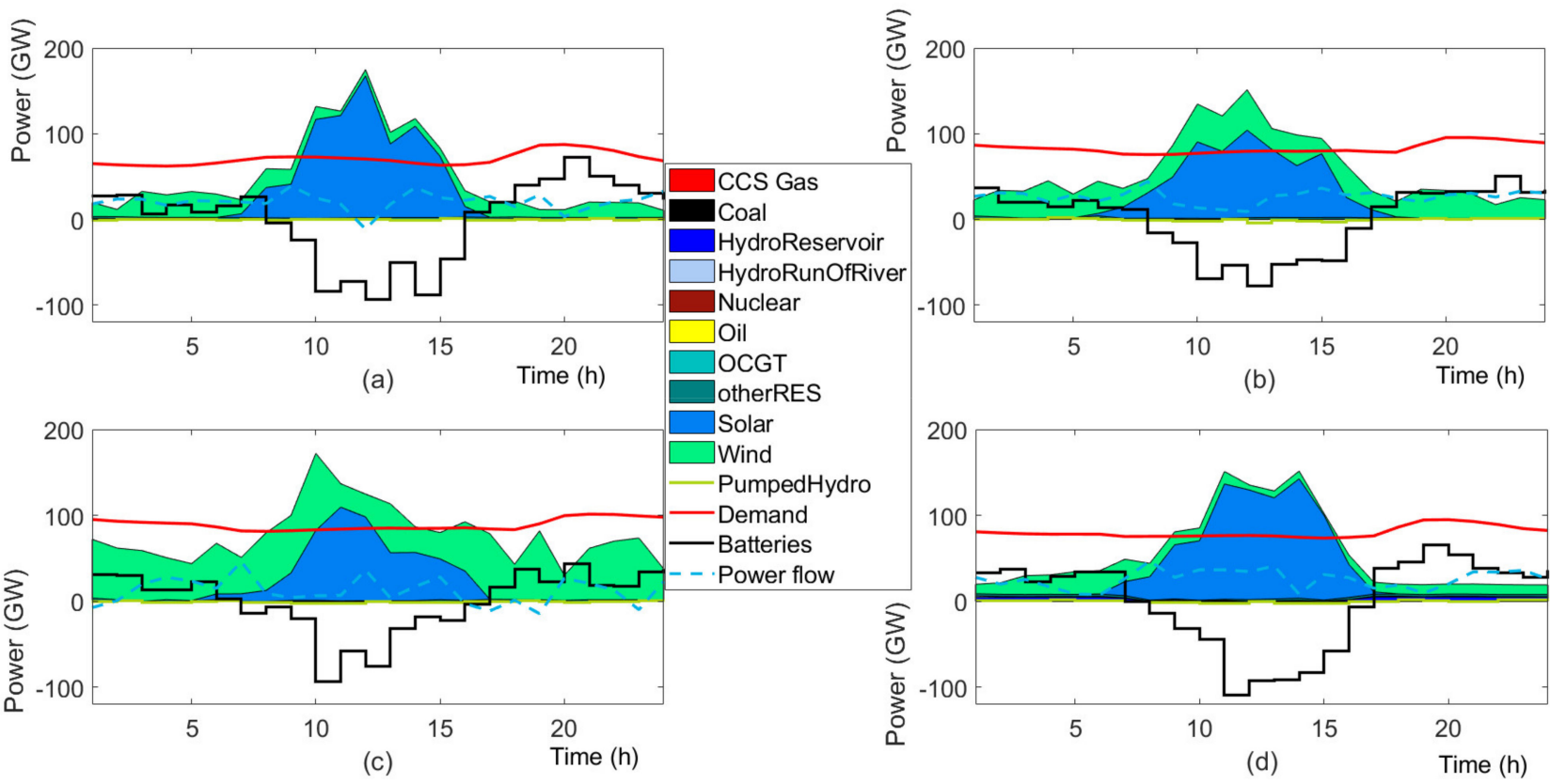

Figure 15. Generation mix in region ER according to scenario node 8, with the availability of storage, in four typical days. Figure (a) reports a typical day within the period January-March, (b) a typical day within April-June, (c) a typical day within July-September, and (d) a typical day within October-December. 

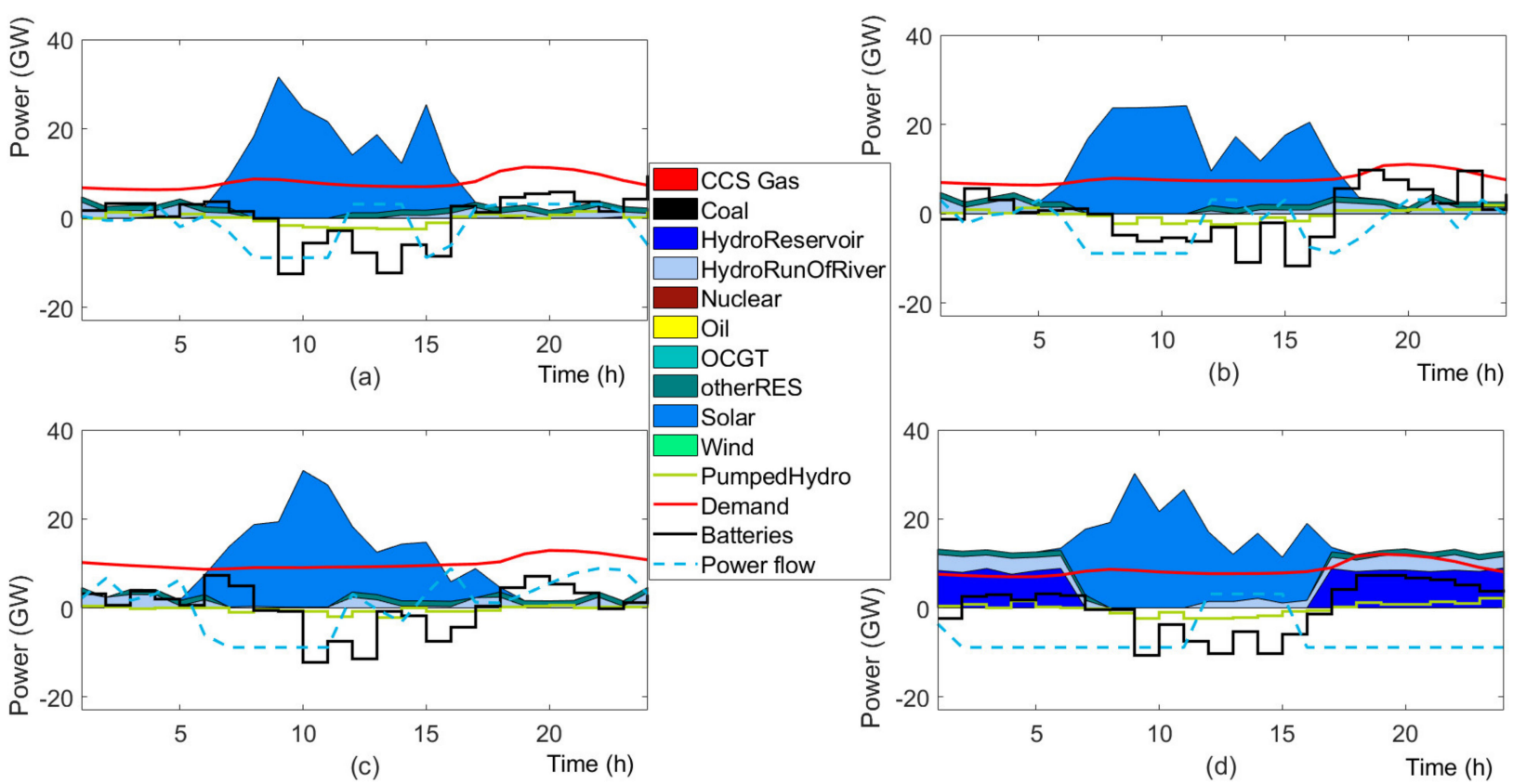

Figure 16. Generation mix in region NER according to scenario node 8 with the availability of storage, in four typical days. Figure (a) reports a typical day within the period January-March, (b) a typical day within April-June, (c) a typical day within July-September, and (d) a typical day within October-December.
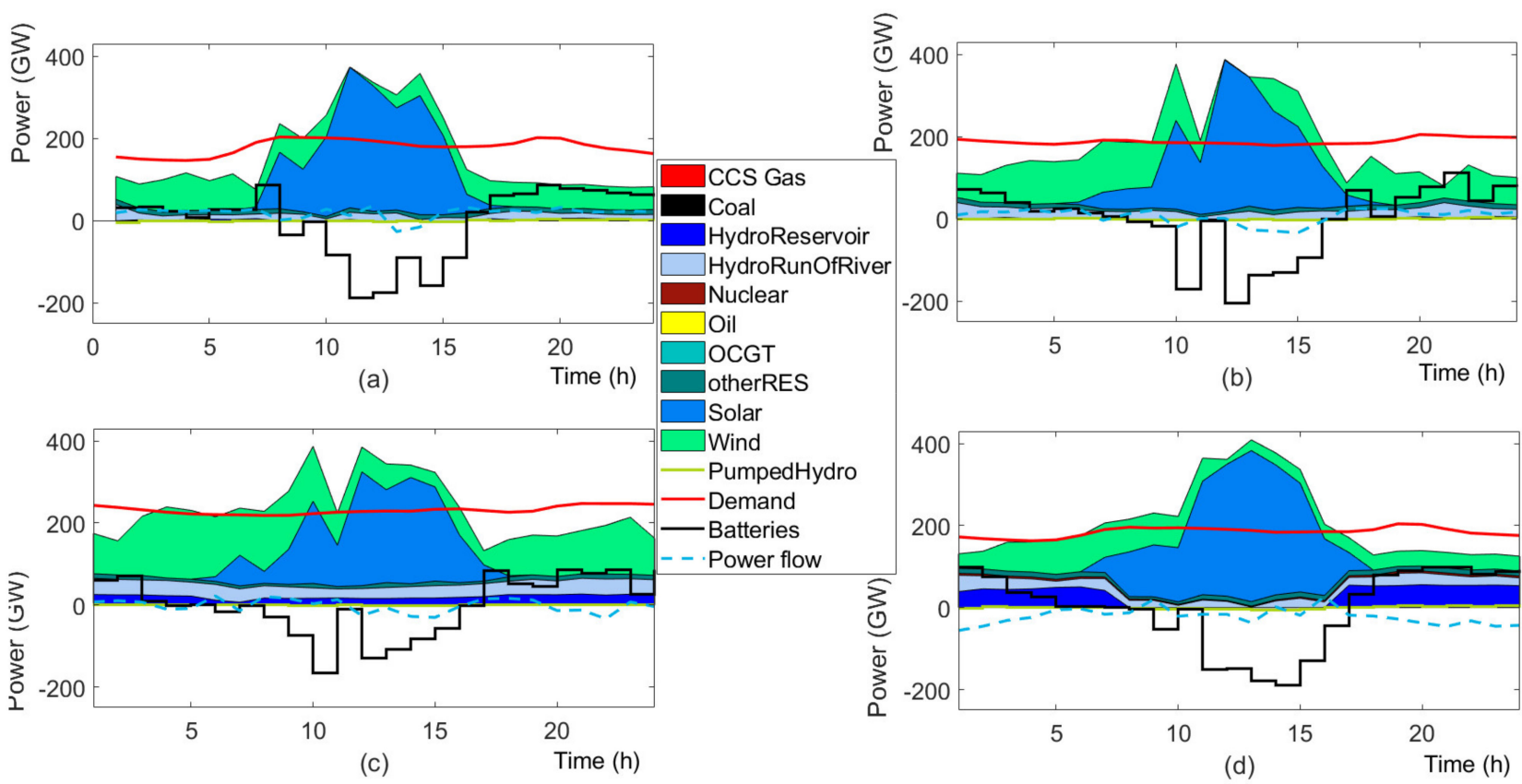

Figure 17. Generation mix in region NR according to scenario node 8 with the availability of storage, in four typical days. Figure (a) reports a typical day within the period January-March, (b) a typical day within April-June, (c) a typical day within July-September, and (d) a typical day within October-December.

The value of storage in decreasing system and line investment cost is demonstrated in Figures 20-23. Figure 20 shows how the accumulated line investment cost is consistently lower in all scenarios when energy storage is included as a candidate option. Figure 22 highlights, for each scenario node, the achieved accumulated line cost difference between the cases with storage and without storage. The optimal operation cost in the last epoch is reported in Figure 21 and the operation cost difference between the two cases is in Figure 23. 
The reduction in operation cost is substantial in the last epoch, and it achieves a reduction of up to $96 \%$ compared to the cost experienced without storage.
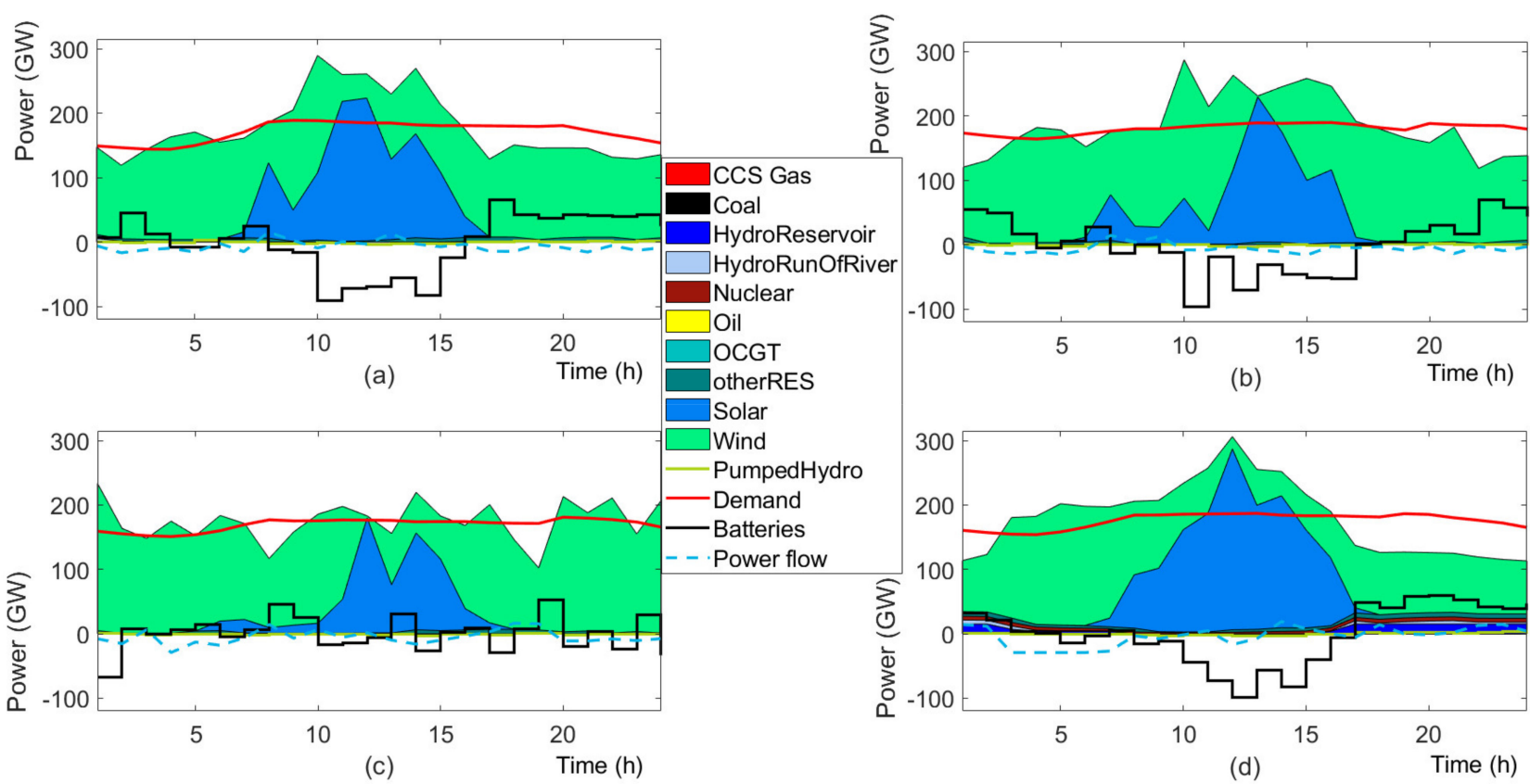

Figure 18. Generation mix in region SR according to scenario node 8 with the availability of storage, in four typical days. Figure (a) reports a typical day within the period January-March, (b) a typical day within April-June, (c) a typical day within July-September, and (d) a typical day within October-December.
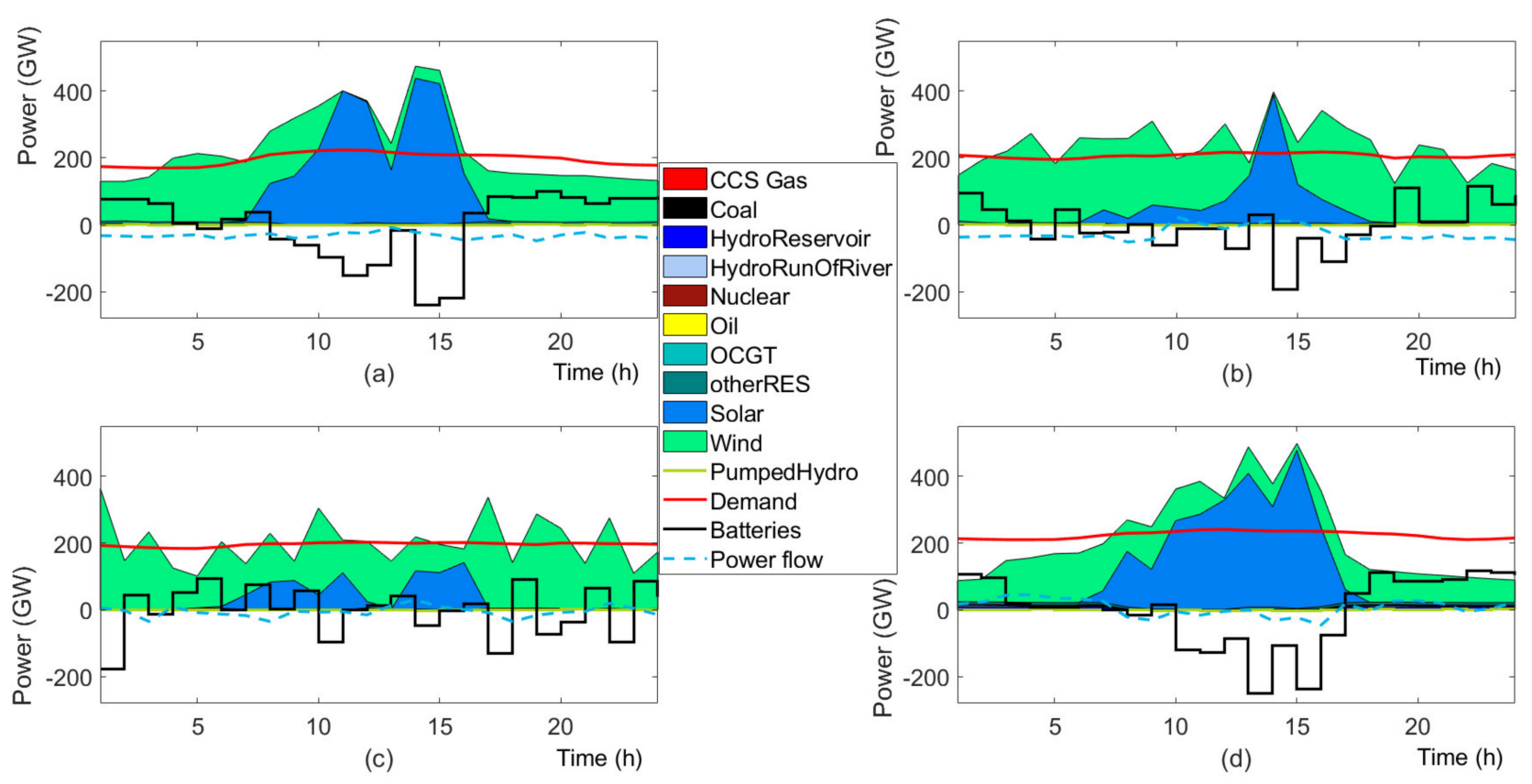

Figure 19. Generation mix in region WR according to scenario node 8 with the availability of storage, in four typical days. Figure (a) reports a typical day within the period January-March, (b) a typical day within April-June, (c) a typical day within July-September, and (d) a typical day within October-December. 


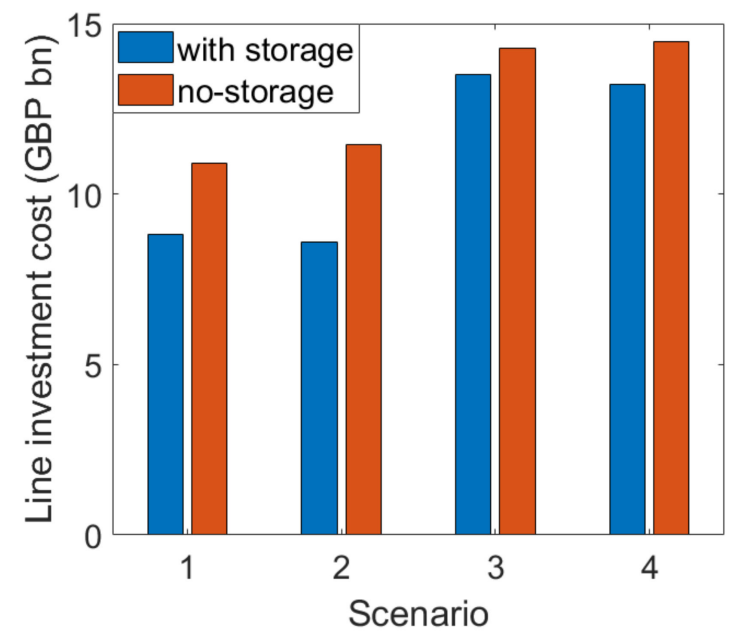

Figure 20. Optimal accumulated line investment cost per scenario for the stochastic studies with and without storage.

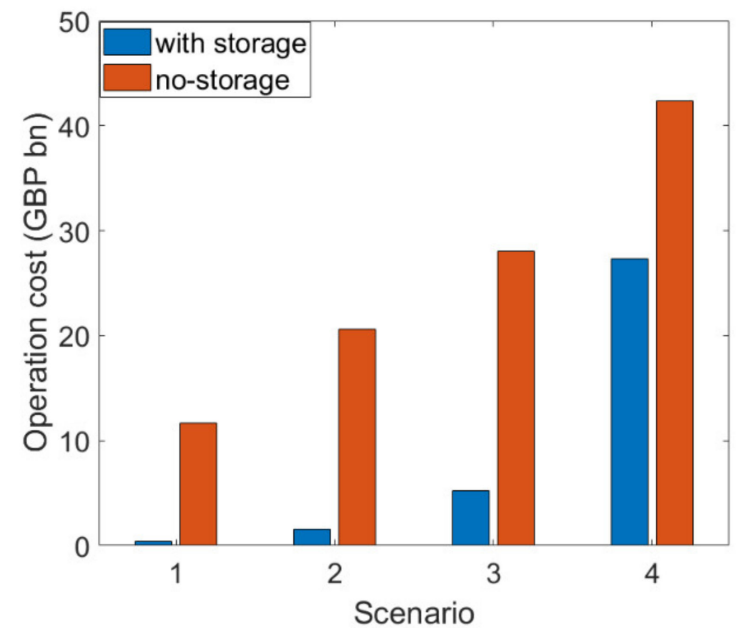

Figure 21. Optimal operation cost in the last epoch per scenario for the stochastic studies with and without storage.

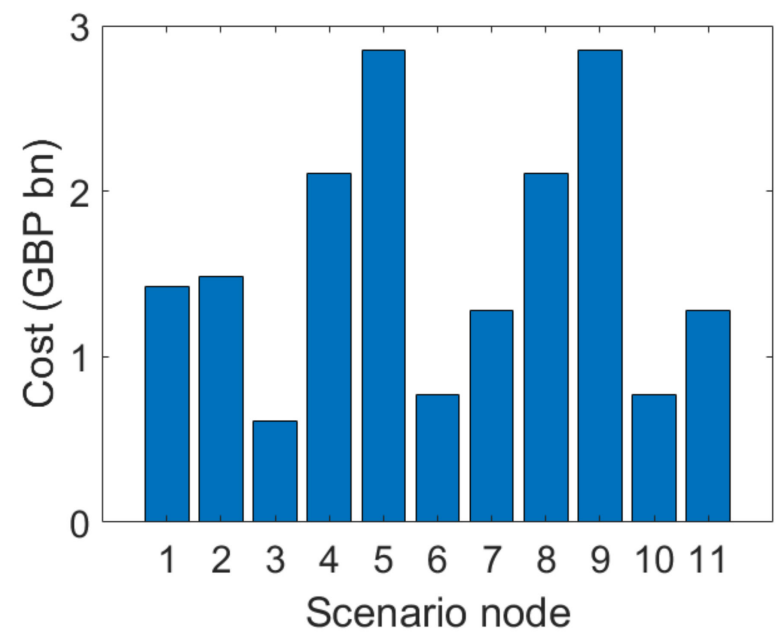

Figure 22. Difference between the optimal accumulated line investment costs for the stochastic studies with and without storage, for every scenario-tree node. 


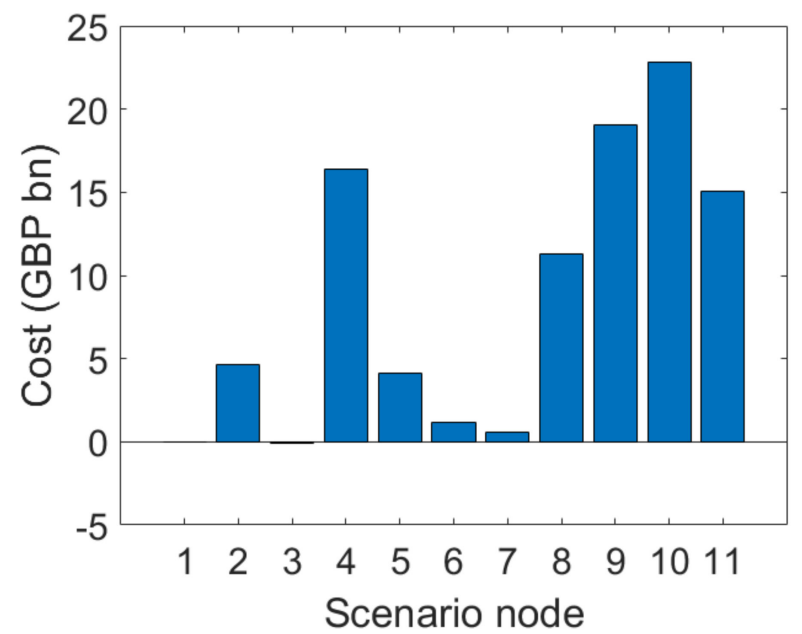

Figure 23. Difference between the optimal operation costs for the stochastic studies with and without storage, per scenario-tree node.

\subsubsection{Stochastic versus Deterministic Planning}

So far, the above studies have involved running a stochastic optimization study (i.e., use of a scenario tree). These results of the stochastic framework have been compared with the results of a deterministic dynamic formulation (i.e., the individual scenarios comprising the scenario tree are solved separately). The investment costs in line reinforcement and energy storage for the deterministic and the stochastic formulations are compared in Figure 24, which shows that the stochastic formulation tends to delay investment decisions in such inflexible assets until strictly required and new information on the system is revealed. On the other hand, some deterministic scenarios, having a fixed view of the future, tend to overinvest in the very beginning, as opposed to a more conservative approach to investment taken by the stochastic optimization approach. The results underline the prevalent risk of overinvestment that is related to deterministic planning frameworks, which by default do not capture uncertainty. Whereas, under a stochastic approach, planning is conducted on the basis of a scenario tree, as opposed to a fixed path, and this allows changes in investment depending on how uncertainty resolves. Thus, stochastic optimization tends to prevent very high investments happening in the very beginning, when the level of uncertainty is at its highest point.

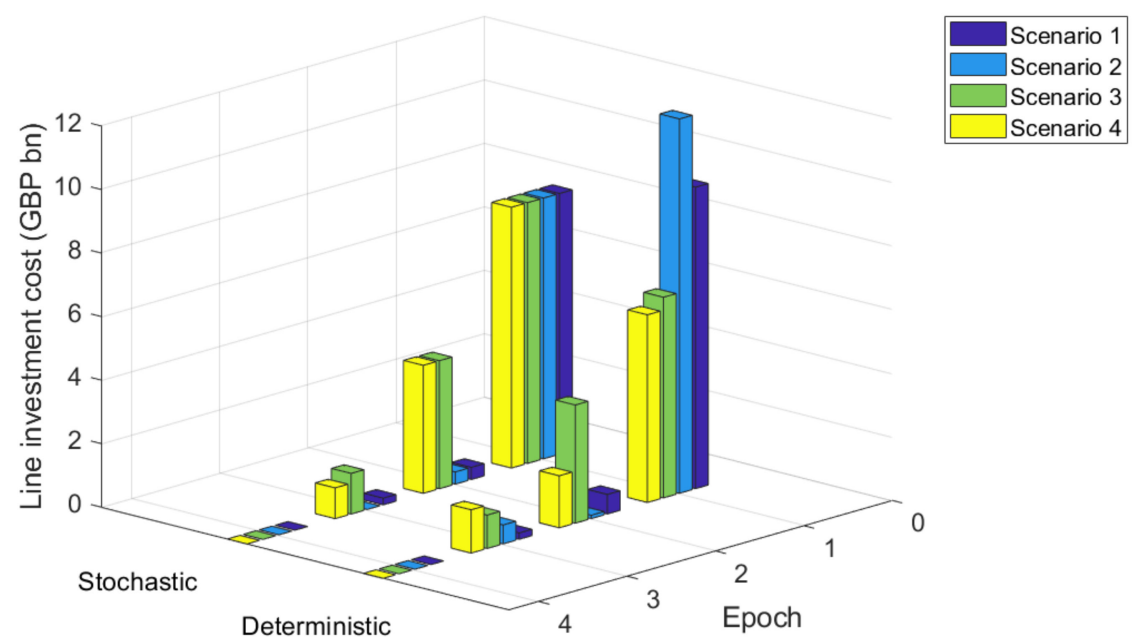

Figure 24. Optimal investment cost in line reinforcement for the deterministic and stochastic formulations for each epoch and scenario. 


\section{Conclusions and Future Work}

This paper presents an application of an advanced mixed integer linear stochastic optimization problem for the network expansion planning of the transmission system in India over the period from the 1 January 2020 until the 1 January 2060. Given the increase in electricity demand as well as in renewables penetration, the planner can invest in conventional network reinforcement, involving building electricity transmission lines, as well as in energy storage. The model has been solved via the use of the Nested Benders decomposition methodology.

The results demonstrate the pivotal role that energy storage can play in enabling the high penetration of renewables in the India's electricity system. It is shown that a considerable amount of energy storage is expected to be invested so as to enable the large-scale integration of wind, solar PV and other renewable sources of electricity. The key metric derived from the analysis is that of the Option Value of storage; the economic benefit of planning with energy storage, under uncertainty, is quantified as Option Value, and it is shown to be approximately GBP $13 \mathrm{bn}$. The Option Value of energy storage is driven by the ability of the technology to optimize system operation by enabling more lowmarginal-cost generation as well as its ability to offer investment flexibility through deferral or displacement of expensive conventional reinforcement, thereby allowing reduction of conventional reinforcement costs. Lastly, the integration of energy storage as an investment option in a multi-stage stochastic planning formulation enables the planner to reduce exposure to the risk of overinvestment, in the event of an adverse scenario occurring.

In terms of future work, the authors aim to apply the proposed framework to case studies covering other countries, as well as interconnected systems of several countries or on a continental level. The current work could also be expanded to evaluate the effect of planning with different types of energy storage technologies or other smart investment options, such as demand-side response and FACTS. Finally, the authors are interested in integrating other energy vectors, such as natural gas, into the planning model, which would also involve the development of new decomposition methodologies.

Author Contributions: Data curation, S.G. and A.J.; Formal analysis, S.G., S.B. and G.S.; Investigation, S.G., P.F., R.B. and J.M.; Methodology, S.B., A.M. and G.S.; Resources, A.J. and R.B.; Software, S.B., P.F. and A.M.; Writing-original draft, S.G. All authors have read and agreed to the published version of the manuscript.

Funding: This work was supported by the Joint UK-India Clean Energy Centre (JUICE) project.

Institutional Review Board Statement: Not applicable.

Informed Consent Statement: Not applicable.

Data Availability Statement: Not applicable.

Conflicts of Interest: The authors declare that there are no conflict of interest.

\section{Nomenclature}

Sets and indices

$\Omega_{B}$

$\Omega_{G}$

$\Omega_{G}^{H_{r r}}$

$\Omega_{H}$

$\Omega_{H}^{T}$

$\Omega_{L}$

$\Omega_{L}^{A C}$

$\Omega_{M}$

$\Omega_{E}$
Set of all demand blocks, indexed $b$

Set of all generation units, indexed $g$

Set of all hydro run of river generation units

Set of all hydro reservoir generation units

Set of all storage units, indexed $h$

Set of candidate storage technologies, indexed $h$

Set of all transmission lines, indexed $l$

Set of all AC transmission lines

Set of all scenario-tree nodes, indexed $m$

Set of all stages, indexed by $e$ 


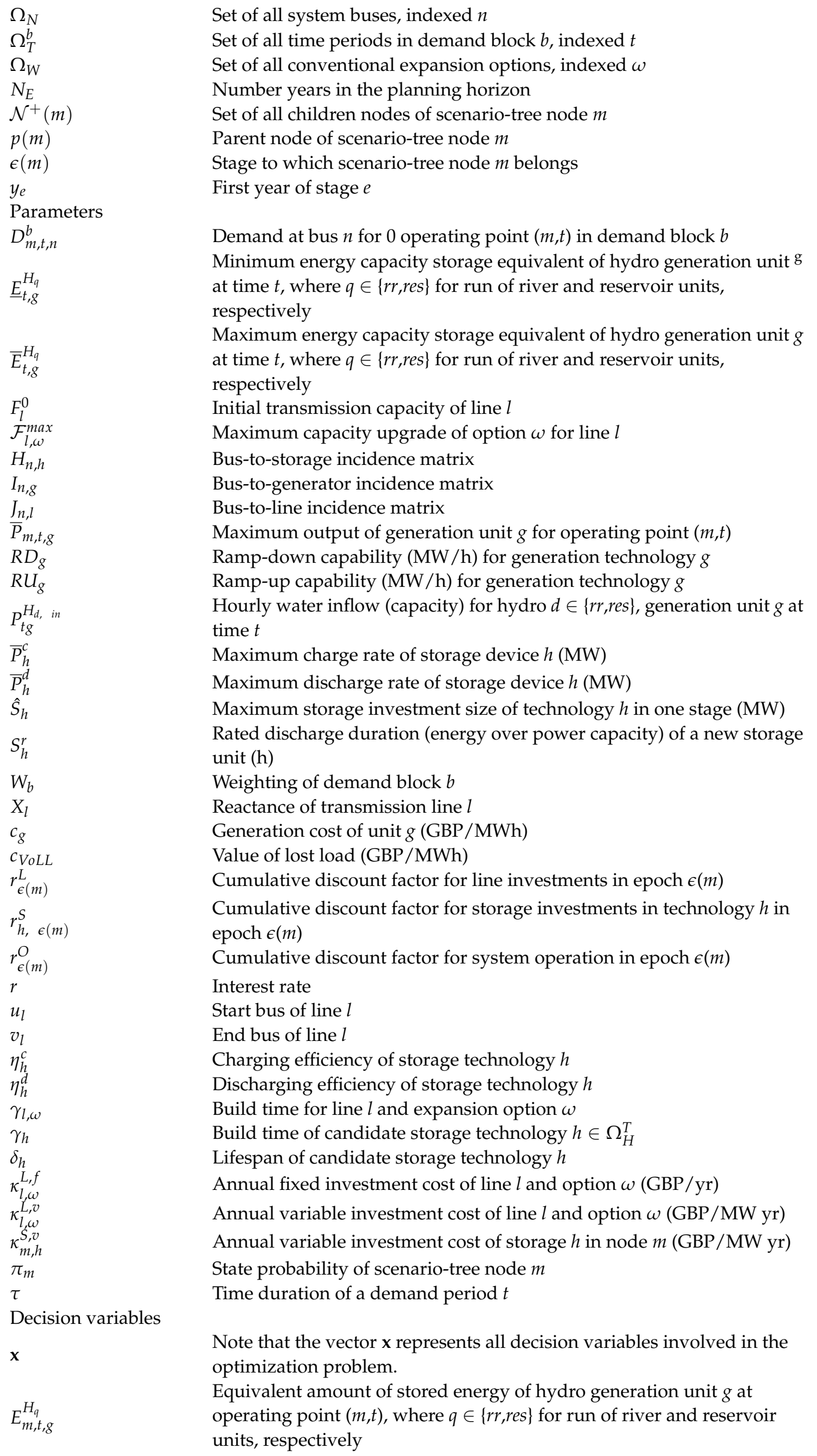




$F_{m, l, \omega}^{I}$
$\widetilde{F}_{m, l}^{I}$
$P_{m, t, g}$
$P_{m, t, h}^{c}$
$P_{m, t, h}^{d}$
$P_{m, t, g}^{H_{q}}$
$P_{m, t, g}^{H_{q,}, h^{2}}$
$S_{m, h}^{I}$
$\widetilde{S}_{m, h}^{I}$
$d_{m, t, n}$
$f_{m, t, l}$

$x_{m}^{I}$

$y_{m}$
$\beta_{m, l, \omega}^{I}$
$\theta_{m, t, n}$
$\sigma_{m, t, h}$

Transmission line capacity upgrade for line $l$ using option $\omega$ at node $m$ Aggregate transmission line capacity upgrades of line $l$ at node $m$ Output of generation unit $g$ at operating point $(m, t)$ Charging power of storage device $h$ at operating point $(m, t)$ Discharging power of storage device $h$ at operating point $(m, t)$ Output of hydro generation unit $g$ at operating point $(m, t)$, where $q \in$ $\{r r, r e s\}$ for run of river and reservoir units, respectively

Water shed equivalent of hydro generation unit $g$ for $q \in\{r r, r e s\}$ at operating point $(m, t)$

Decision variable modelling investment in storage asset $h$ at node $m$ Aggregate storage capacity investment for asset $h$ at node $m$ Demand curtailment at bus $n$ and operating point $(m, t)$

Power flow on line $l$ at operating point $(m, t)$ Decision vector coupling investments in node $m$ with those in its parent node $p(m)$, where $I \in\left\{\beta, c, F, S, S^{R}\right\}$ for line investment decisions, transmission capacity investment, aggregate transmission capacity investment, storage investment decisions, and storage decommissioning decisions, respectively

Linear combination of $x_{m}^{I}$, where $I \in\left\{\beta, c, F, S, S^{R}\right\}$ for line investment decisions, transmission capacity investment, aggregate transmission capacity investment, storage investment decisions, and storage decommissioning decisions, respectively Binary decision variable modelling investment in transmission capacity in line $l$ using option $\omega$ at node $m$ Voltage angle at bus $n$ at operating point $(m, t)$ State of charge of storage device $h$ at operating point $(m, t)$

\section{References}

1. Krishnan, R.; Sanjay, J.; Gnanaseelan, C.; Mujumdar, M.; Kulkarni, A.; Chakraborty, S. Assessment of Climate Change over the Indian Region: A Report of the Ministry of Earth Sciences (MoES), Government of India. 2020. Available online: https: / / link.springer.com/book/10.1007\%2F978-981-15-4327-2 (accessed on 1 November 2021). [CrossRef]

2. Available online: https://unfccc.int/process-and-meetings/the-paris-agreement/the-paris-agreement (accessed on 1 November 2021).

3. Available online: https://www.eia.gov/international/content/analysis/countries_long/India/india.pdf (accessed on 1 November 2021).

4. Available online: https:/ / saubhagya.gov.in/ (accessed on 1 November 2021).

5. The Times of India. Power distribution plan soon to ensure 24X7 electricity supply for all. 16 July 2019; Live Mint. Govt's 100-day plan aims to re-energize India's power sector. The Times of India, 12 June 2019, p. 6.

6. International Energy Agency. World Energy Outlook 2019, Annexes; BP Statistical Review of World Energy 2019; International Energy Agency: Paris, France, 2019.

7. Available online: https://niti.gov.in/writereaddata/files/175-GW-Renewable-Energy.pdf (accessed on 1 November 2021).

8. Available online: https:// data.worldbank.org/indicator/EN.ATM.CO2E.PC?locations=IN (accessed on 1 November 2021).

9. Strbac, G.; Konstantelos, I.; Pollitt, M.; Green, R. Report for the UK National Infrastructure Commission: Delivering Future-Proof Energy Infrastructure. Available online: https://assets.publishing.service.gov.uk/government/uploads/system/uploads/ attachment_data/file/507256/Future-proof_energy_infrastructure_Imp_Cam_Feb_2016.pdf (accessed on 30 January 2020).

10. Available online: https://www.iea.org/reports/india-2020 (accessed on 1 November 2021).

11. Giannelos, S.; Konstantelos, I.; Strbac, G. Option value of dynamic line rating and storage. In Proceedings of the 2018 IEEE International Energy Conference (ENERGYCON), Limassol, Cyprus, 3-7 June 2018; pp. 1-6.

12. Giannelos, S.; Konstantelos, I.; Strbac, G. Option Value of Demand-Side Response Schemes Under Decision-Dependent Uncertainty. IEEE Trans. Power Syst. 2018, 33, 5103-5113. [CrossRef]

13. Konstantelos, I.; Giannelos, S.; Strbac, G. Strategic Valuation of Smart Grid Technology Options in Distribution Networks. IEEE Trans. Power Syst. 2017, 32, 1293-1303. [CrossRef]

14. Andrychowicz, M. The Impact of Energy Storage along with the Allocation of RES on the Reduction of Energy Costs Using MILP. Energies 2021, 14, 3783. [CrossRef]

15. Andrychowicz, M. RES and ES Integration in Combination with Distribution Grid Development Using MILP. Energies 2021, 14, 383. [CrossRef]

16. Laha, P.; Chakraborty, B. Cost optimal combinations of storage technologies for maximizing renewable integration in Indian power system by 2040: Multi-region approach. Renew. Energy 2021, 179, 233-247. [CrossRef] 
17. Frerk, M. Investing for Net Zero in the Face of Uncertainty: Real Options and Robust Decision-Making; The Oxford Martin School Programme on Integrating Renewable Energy; Working Paper; March 2021. Available online: https://www.oxfordmartin. ox.ac.uk/downloads/academic/Oxford-strategic-investment-150321.pdf (accessed on 1 November 2021).

18. Giannelos, S.; Djapic, P.; Pudjianto, D.; Strbac, G. Quantification of the Energy Storage Contribution to Security of Supply through the F-Factor Methodology. Energies 2020, 13, 826. [CrossRef]

19. Pudjianto, D.; Aunedi, M.; Djapic, P.; Strbac, G. Whole-Systems Assessment of the Value of Energy Storage in Low-Carbon Electricity Systems. IEEE Trans. Smart Grid 2014, 5, 1098-1109. [CrossRef]

20. Alvarado, D.; Moreira, A.; Moreno, R.; Strbac, G. Transmission Network Investment with Distributed Energy Resources and Distributionally Robust Security. IEEE Trans. Power Syst. 2019, 34, 5157-5168. [CrossRef]

21. Nieto, A.; Vita, V.; Maris, T.I. Power quality improvement in power grids with the integration of energy storage systems. Int. J. Eng. Res. Technol. 2016, 5, 438-443.

22. Papadaskalopoulos, D.; Pudjianto, D.; Strbac, G. Decentralized Coordination of Microgrids with Flexible Demand and Energy Storage. IEEE Trans. Sustain. Energy 2014, 5, 1406-1414. [CrossRef]

23. Agamah, S.; Ekonomou, L. Energy storage system scheduling for peak demand reduction using evolutionary combinatorial optimization. Sustain. Energy Technol. Assess. 2017, 23, 73-82.

24. Available online: https://www.iea.org/commentaries/india-is-going-to-need-more-battery-storage-than-any-other-countryfor-its-ambitious-renewables-push (accessed on 1 November 2021).

25. Giannelos, S.; Konstantelos, I.; Strbac, G. Investment Model for Cost-effective Integration of Solar PV Capacity under Uncertainty using a Portfolio of Energy Storage and Soft Open Points. In Proceedings of the 2019 IEEE Milan PowerTech, Milan, Italy, 23-27 June 2019. [CrossRef]

26. Binder, W.R.; Paredis, C.J.J.; Garcia, H.E. The Value of Flexibility in the Design of Hybrid Energy Systems: A Real Options Analysis. IEEE Power Energy Technol. Syst. J. 2017, 4, 74-83. [CrossRef]

27. Kauppinen, L.; Siddiqui, A.S.; Salo, A. Investing in Time-to-Build Projects with Uncertain Revenues and Costs: A Real Options Approach. IEEE Trans. Eng. Manag. 2018, 65, 448-459. [CrossRef]

28. Moreira, A.; Pozo, D.; Street, A.; Sauma, E.; Strbac, G. Climate-aware generation and transmission expansion planning: A three-stage robust optimization approach. Eur. J. Oper. Res. 2021, 295, 1099-1118. [CrossRef]

29. Moreira, A.; Strbac, G.; Fanzeres, B. An ambiguity averse approach for transmission expansion planning. In Proceedings of the 2019 IEEE Milan PowerTech, Milan, Italy, 23-27 June 2019. [CrossRef]

30. Moreira, A.; Strbac, G.; Moreno, R.; Street, A.; Konstantelos, I. A Five-Level MILP Model for Flexible Transmission Network Planning Under Uncertainty: A Min-Max Regret Approach. IEEE Trans. Power Syst. 2018, 33, 486-501. [CrossRef]

31. Falugi, P.; Konstantelos, I.; Strbac, G. Planning with Multiple Transmission and Storage Investment Options Under Uncertainty: A Nested Decomposition Approach. IEEE Trans. Power Syst. 2018, 33, 3559-3572. [CrossRef]

32. Conejo, A.J.; Baringo, L.; Kazempour, S.J.; Siddiqui, A.S. Investment in Electricity Generation and Transmission: Decision Making under Uncertainty; Springer: New York, NY, USA, 2016.

33. Long Term Electricity Demand Forecasting; Tech. Rep.; Central Electricity Authority, Ministry of Power, Government of India: New Delhi, India, 2019. Available online: https:/ / cea.nic.in/old/reports/others/planning/pslf/Long_Term_Electricity_Demand_ Forecasting_Report.pdf (accessed on 28 August 2021).

34. Pradhan, G.B.; Singhal, A.K.; Bakshi, A.S.; Iyer, M.K. Determination of Transmission Tariff for 2014-15 Tariff Period; Petition No. 173/TT/2016; Central Electricity Regulatory Commission: New Delhi, India, 2017. Available online: https://cercind.gov.in/2017 / orders/173_TT.pdf (accessed on 28 August 2021). 